\title{
On Counting the Population Size
}

\author{
Petra Berenbrink $^{1} \quad$ Dominik Kaaser ${ }^{1} \quad$ Tomasz Radzik $^{2}$ \\ 1 Universität Hamburg \\ Hamburg, Germany \\ \{petra.berenbrink,dominik.kaaser\}@uni-hamburg.de \\ 2 King's College London \\ London, UK \\ tomasz.radzik@kcl.ac.uk
}

\begin{abstract}
We consider the problem of counting the population size in the population model. In this model, we are given a distributed system of $n$ identical agents which interact in pairs with the goal to solve a common task. In each time step, the two interacting agents are selected uniformly at random. In this paper, we consider so-called uniform protocols, where the actions of two agents upon an interaction may not depend on the population size $n$. We present two population protocols to count the size of the population: protocol ApProximate, which computes with high probability either $\lfloor\log n\rfloor$ or $\lceil\log n\rceil$, and protocol COUNTEXACT, which computes the exact population size in optimal $\mathrm{O}(n \log n)$ interactions, using $\tilde{\mathrm{O}}(n)$ states. Both protocols can also be converted to stable protocols that give a correct result with probability 1 by using an additional multiplicative factor of $\mathrm{O}(\log n)$ states.
\end{abstract}

\section{Introduction}

In this paper we consider the problem of counting the population size in the probabilistic population model. The model was introduced in [5] to model distributed systems of resource-limited mobile agents, which interact with each other in order to solve a common task. The computation of a probabilistic population protocol can be viewed as a sequence of pairwise interactions of randomly chosen agents. In each interaction, the two participating agents observe each others' states and update their own state according to a transition function common to all agents.

In this setting, we are interested in so-called uniform population protocols, where the transition function does not depend on the size of the population, so it can be applied to any population size. In the original definition of population protocols in [5], each agent is a copy of the same finite state machine. Such a protocol is by definition uniform, as its state space has constant size (independent of the size of the population). However, more recent results have shown that many problems can be solved much faster if agents are equipped with a number of states that grows with the population size. For example, a simple protocol with a constant number of states solves the majority problem in expected $\mathrm{O}\left(n^{2}\right)$ interactions $[17,20]$. On the other hand, a number of protocols have been proposed which solve the majority problem in $\mathrm{O}(n$ polylog $n)$ interactions using $\mathrm{O}($ polylog $n)$ states $[1,2,12,9]$. These protocols are all non-uniform since their transition functions refer to values which have to be $\Theta(\log n)$. We note that $\Omega(n \log n)$ interactions are required to reach 
a positive constant probability that each agent participates in at least one interaction, so $\Omega(n \log n)$ is a lower bound on the number of interactions of a protocol which solves any nontrivial problem.

Turning to the problem of counting the population size, either exactly or approximately, we naturally have to consider population protocol models with growing memory, where lower bounds on the required number of states depend on the desired counting accuracy. The exact counting of the population size requires $\Omega(n)$ states, while estimating the population size up to a constant factor requires $\Omega(\log n)$ states.

There is a simple and uniform protocol for exact population counting, which completes in expected $\Theta\left(n^{2}\right)$ interactions and uses $\Theta\left(n^{2}\right)$ states: the agents start with one token each and keep combining the tokens into bags, propagating at the same time the maximum size of a bag and using that maximum as their current output. The transition function of this protocol does not refer in any way to (any estimate of) the size of the population.

In this paper we are interested in uniform population counting protocols which run in $\mathrm{O}(n$ polylog $n)$ interactions and use a small number of states (relatively to the lower bounds). We present new protocols for exact and approximate population counting, which use a significantly smaller number of states than the previously best protocols shown in $[13,14]$. Our protocols have also further desirable properties. Our exact protocol is the first protocol for this problem which completes the computation within asymptotically optimal $\mathrm{O}(n \log n)$ interactions. A variant of our approximate protocol is the first $\mathrm{O}($ polylog $n)$-state, $\mathrm{O}(n$ polylog $n)$-interaction protocol always converging to a value which is within a constant factor from the exact size of the population. To give formal statements of the previous results and our new results, we have to introduce first some details of the model and the way the efficiency of population protocols is measures.

\subsection{Computation Model}

The computation model is a population of $n$ agents which are capable of performing local computations. A population protocol is specified by a state space $Q$, an output domain $O$, a transition function $\delta: Q \times Q \rightarrow Q \times Q$, and an output function $\omega: Q \rightarrow O$. Each agent has a state $q \in Q$, which is updated during interactions. The current output of an agent in state $q$ is $\omega(q)$. The current configuration of the system is the vector from $Q^{n}$ with the current states of the agents. The computation of a population protocol is a sequence of pairwise interactions of agents. In every time step, a probabilistic scheduler selects independently and uniformly at random a pair of agents for interaction, with the first agent called the initiator and the second the responder. During the interaction the agents update their states by applying the transition function $\delta$. Such an update is denoted by $(x, y) \rightarrow\left(x^{\prime}, y^{\prime}\right)$, where $(x, y)$ refers to the states of the agents before the interaction and $\left(x^{\prime}, y^{\prime}\right)=\delta(x, y)$ to the states after the interaction.

A given problem which we want to solve by population protocols specifies the set of initial (input) configurations, the output domain $O$, and the desired (output) configurations for given input configurations. For the exact population counting problem, all agents are initially in the same state $q_{0}$ and the output domain is the set of positive integers. A desired configuration is when all agents output correctly the (exact) size of the population. In the leader election problem, which arises as a sub-problem in many population protocols, including in the protocols for the population counting problem, all agents start with the same initial state and the output domain is $O=\{$ leader,follower $\}$. A desired configuration is when exactly one agent outputs that it is the leader.

The following two definitions are commonly used to capture important aspects of the time efficiency of population protocols. The convergence time $T_{C}$ of an execution of a protocol is the number of interactions until the system enters a desired configuration and never leaves the set of 
desired configurations again. The stabilization time $T_{S}$ of an execution of a protocol is the number of interactions until the system enters a desired stable configuration, meaning that starting from this configuration no sequence of pairwise interactions can take the system outside of the set of desired configurations. We always have $T_{C} \leq T_{S}$ for any execution of a protocol, but the stabilization time may be strictly greater than the convergence time. A population protocol is always correct (or stable), if for each initial configuration the computation reaches a correct stable configuration with probability 1, or w.h.p. correct, if there is a small (of the order of $n^{-\Omega(1)}$ ), but positive, probability that the system settles with an incorrect output or does not settle at all. We say that a protocol converges (resp. stabilizes) in $T(n)$ time w.h.p. (resp. in expectation), if $T_{C}$ (resp. $T_{S}$ ) is at most $T(n)$ w.h.p. ${ }^{1}$ (resp. in expectation).

The second measure of efficiency of population protocols is the required number of states. This is a straightforward notion for simple protocols with constant number of states. For more complex protocols, transition functions are usually described by pseudo-codes, which refer to variables. The state space of a protocol which is defined in this way is the Cartesian product of the ranges of the variables. If we consider all possible executions of a uniform protocol, then the ranges of some variables may be very large in terms of $n$, potentially infinite. We are, however, interested in bounds on the ranges of the variables (and thus bounds on the whole state space) that hold w.h.p.

A population protocol is called uniform if the same transition function is used for all population sizes [7]. Uniformity of a protocol is thus a desired property since such protocols can be applied without knowing the size of the population in advance. The original model from $[4,5]$ is uniform since the number of states of an agent is constant.

\subsection{Related Work}

It is shown in $[4,5]$ that with a constant number of states all semilinear predicates (which include, e.g., the majority predicate), can be computed in expected $\mathrm{O}\left(n^{2} \log n\right)$ stabilization time. Recently Kosowski and Uznanski [19] have shown constant-state protocols for computing the semilinear predicates and for electing a leader, which w.h.p. have $\mathrm{O}(n$ polylog $n)$ convergence time. Achieving fast $\mathrm{O}(n$ polylog $n)$ stabilization time for the majority and leader election problems requires a growing state space, as $\mathrm{O}\left(n^{2-\varepsilon}\right)$ stabilization time is not possible with a constant number of states $[16,1]$.

There are a number of protocols for the majority problem and the leader election problem with $\mathrm{O}(n$ polylog $n)$ stabilization time and $\mathrm{O}(\operatorname{polylog} n)$ states $[3,1,12,2,9]$, but they all are non-uniform. The transition functions of these protocols depend on $n$ as they use values $\Omega(\log n)$ or $\Omega(\log \log n)$. For example, each agent may be counting its interactions (which are uniform updates, not dependent on $n$ ) and progress to the next phase of the computation when its counter reaches $\log n$ (a non-uniform update).

While the computational power of (uniform) constant-space population protocols is well understood by now, less is known about the power of uniform protocols which allow the state space to grow with the size of population. Doty et al. [14] show a protocol for the exact population counting problem which has $\mathrm{O}(n \log n \log \log n)$ stabilization time and uses $\mathrm{O}\left(n^{60}\right)$ states, w.h.p. and in expectation. They also formalize the notion of uniform population protocols, modeling the agents as copies of the same Turing machine, but describe their protocols using pseudo-codes and take the size of the state space as the product of the ranges of the variables.

Doty and Eftekhari [13] consider the problem of estimating the size of population within a constant factor, which they view as the problem of computing $\log n \pm \mathrm{O}(1)$. They show a protocol which w.h.p.

${ }^{1}$ The expression with high probability (w.h.p.) refers to a probability of at least $1-n^{-\Omega(1)}$. 
has $\mathrm{O}\left(n \log ^{2} n\right)$ convergence time and uses $\mathrm{O}\left(\log ^{7} n \log \log n\right)$ states. Their protocol is not always correct and they left open the question to design a protocol which uses expected $\mathrm{O}(n \operatorname{polylog} n)$ interactions and $\mathrm{O}(\operatorname{polylog} n)$ states and computes $\log n \pm \mathrm{O}(1)$ with probability 1 . An earlier work by Alistarh et al. [1] includes a uniform protocol which uses $\mathrm{O}(n \log n)$ interactions and $\mathrm{O}(\log n)$ states in expectation to compute an integer which w.h.p. is between $c_{1} \log n$ and $c_{2} \log n$, for some constants $0<c_{1}<1<c_{2}$. This can be viewed as approximating the population size within a polynomial factor.

\subsection{Our Results}

In this paper we present and analyze two uniform protocols, protocol Approximate for approximating the population size within constant factors, and protocol CoUNTEXACT for computing the exact number of agents in the population. Unless stated otherwise we assume that all agents have to output the population size or its approximation. The theorems below summarize the performance of our protocols.

Theorem 1. Protocol Approximate is uniform and outputs w.h.p. either $\lfloor\log n\rfloor$ or $\lceil\log n\rceil$.

1) It converges in at most $\mathrm{O}\left(n \log ^{2} n\right)$ interactions using $\mathrm{O}(\log n \cdot \log \log n)$ states, w.h.p.

2) A variant of the protocol stabilizes in $\mathrm{O}\left(n \log ^{2} n\right)$ interactions using at most $\mathrm{O}\left(\log ^{2} n \cdot \log \log n\right)$ states, w.h.p.

3) Stabilization can also be achieved w.h.p. in $\mathrm{O}\left(n \log ^{2} n\right)$ interactions using $\mathrm{O}(\log n \cdot \log \log n)$ states, if not all but only $n-\log n$ agents need to output the result.

Theorem 2. The protocol COUnTEXACT is uniform and outputs the exact population size $n$. It stabilizes in $\mathrm{O}(n \log n)$ interactions and uses $\tilde{\mathrm{O}}(n)$ states, w.h.p. ${ }^{2}$

Our protocols improve considerably the time and space bounds of the previous work [14] and [13]. Moreover, our approximate protocol is stable, answering the open question posed in [13], and it calculates a tighter approximation converging to $\lfloor\log n\rfloor$ or $\lceil\log n\rceil$ instead of $\log n \pm 4.7$ shown in [13]. The stabilization time of our exact counting protocol is asymptotically optimal and its number of states is only a polylog $n$ factor away from the lower bound $n$. We note that in our protocol for exact counting the output value of an agent is a function of the state of this agent, and $n$ is not kept explicitly in the agent's state. This is consistent with the approach in [14].

Our algorithms are based on leader election followed by load balancing phases. A load balancing phase starts with the total load of $M$ tokens in the system and the goal is to relate $M$ to $n$ (the size of the population). If this load balancing phase completes with some agents having zero load, then w.h.p. $M \leq c n$, for a fixed constant $c>1$. On the other hand, if all agents end up with positive load, then we must have $M \geq n$. To achieve good time and space bounds, we have to carefully control the load balancing phases and integrate them with the process of leader election. To achieve stability both of our protocols use an error detection routine and the leader initializes a process testing if the calculated answer is correct or not.

In Section 2 we discuss the auxiliary protocols which we use in our main protocols. We outline our approximate and exact protocols in Sections 3 and 4 , respectively.

\footnotetext{
${ }^{2}$ We define $\tilde{\mathrm{O}}(f(n))=\mathrm{O}(f(n) \cdot \log \mathrm{O}(1)(f(n)))$.
} 


\section{Auxiliary Protocols}

In this section we define auxiliary protocols that we will apply in our protocols: one-way epidemics, the junta process, leader election, and phase clocks.

One-Way Epidemics. The goal of one-way epidemics is to spread an information to all members of the population. The state space of the protocol is $\{0, x\}$ for some $x>0$. Initially at least one agent has the value $x$, which is then spread to all other agents. The transitions are formally defined as $\delta(u, v)=(\max \{u, v\}, v)$. We will refer to one-way epidemics also as broadcast. A natural extension is maximum broadcast, where agents do not only spread one possible value $x$, but instead each agent starts with its own value from the integer interval $[0, x]$. For maximum broadcast, an agent always adopts the maximum, which is covered by the transition rule above as well. The result on one-way epidemics carries over immediately to maximum broadcast. The following result is well-known, see for example [6].

Lemma 3. Let $T_{\mathrm{BC}}$ be the number of interactions required to complete (maximum) broadcast. W.h.p., $T_{\mathrm{BC}}=\mathrm{O}(n \log n)$.

Junta Process. The goal of the junta process $[18,8]$ is to mark $\Theta\left(n^{\varepsilon}\right)$ agents - the $j u n t a$. The state of each agent $v$ in this process is formed by a triplet (level $v$, active , junta $_{v}$ ) $\in \mathbb{N}_{0} \times\{0,1\}^{2}$ initially set to $(0,1,1)$. The idea of the protocol is as follows. If an active agent $v$ interacts with an active agent on the same level it increases its level, otherwise it sets active $v_{v}$ to 0 . Whenever $v$ interacts with an agent on a higher level it sets junta ${ }_{v}$ to 0 . Inactive agents adopt the level of their communication partner if that is higher. The protocol stabilizes when all agents are inactive. The junta is formed by all agents $v$ that reached the maximum level and have their junta $v$ bit set to 1 . The following lemma is shown in [8], here it is adapted to our setting.

Lemma 4. ([8]) Let level ${ }^{*}=\max \left\{\right.$ level $\left._{v}\right\}$ be the maximal level reached by the junta process. All agents become inactive within $\mathrm{O}(n \log n)$ interactions, $\log \log n-4 \leq$ level $^{*} \leq \log \log n+8$, and the number of agents on the maximal level is $\mathrm{O}(\sqrt{n} \cdot \log n)$, w.h.p.

Phase Clocks. The existence of a non-empty junta of size in $\left[1, n^{\varepsilon}\right]$ allows the agents to synchronize themselves via so-called phase clocks $[6,18]$. The phase clocks allow all agents to divide the time in phases of $\Theta(n \log n)$ interactions each. All agents $v$ have a state clock $v$ in $\{0, \ldots, m-1\}$ where $m$ is a constant. Intuitively, these clock states can be seen as the hours on a clock face. The basic idea in every interaction is that the agents always adopt the larger clock state w.r.t. the circular order modulo $m$. Additionally, in order to keep the clock running, the members of the junta proceed one additional step when they interact with another agent on the same clock state. An agent $v$ enters a new phase in interaction $t$ if clock $_{v}$ crosses the boundary between $m-1$ and 0 . In that case we say the phase clock ticks.

For easy access to the phase clocks, we equip each agent $v$ with a variable phase ${ }_{v}$ of constant size that counts the current phase of an agent modulo some constant. Additionally, each agent $v$ has a flag $^{3}$ firstTick $_{v}$. This flag is set to 1 whenever the phase ${ }_{v}$ counter is incremented, and it is set to 0 otherwise.

Let $D_{i}=\left[D_{i}^{\text {start }}, D_{i}^{\text {end }}\right]$ be the interval of interactions in Phase $i$ such that the last agent enters Phase $i$ in interaction $D_{i}^{\text {start }}$ and the first agent leaves Phase $i$ in interaction $D_{i}^{\text {end }}+1$.

${ }^{3}$ Note that for all flags we use FALSE (resp. TRUE) and 0 (resp. 1) interchangeably. 
Lemma 5 ([18]). For any constant $c \geq 0$ we can construct a phase clock using $m=m(c)=\mathrm{O}(1)$ states such that w.h.p. for all phases $1 \leq i \leq \operatorname{poly}(n)$ we have cn $\log n \leq D_{i}^{\text {end }}-D_{i}^{\text {start }} \leq$ cn $\log n+$ $\Theta(n \log n)$.

Leader Election. In [18] the authors present a stable and uniform protocol to perform leader election called LEADER_ELECT. It runs in $\mathrm{O}(\log n)$ phases of $\mathrm{O}(n \log n)$ interactions each.

The process starts with junta election in order to start two nested phase clocks. Agents perform an interaction of the outer phase clock once per phase of the inner phase clock. In the beginning, every agent runs the protocols for the phase clocks, junta election, and leader election in parallel. Whenever an agent encounters another agent on a higher (junta) level, it resets the clocks and the leader election protocol. In that way, all agents eventually run the phase clocks and the leader election process based on the junta on the highest level. The actual leader election process is quite simple: the set of leaders is halved from phase to phase, and the time is measured by the inner phase clock.

For stable leader election the authors of [18] combine their protocol with a slow protocol which is always correct in the following way: the outer phase clock runs only if at least one leader remains in the system. If at some point it counts to $m$, w.h.p. a total of $\Theta\left(n \log ^{2} n\right)$ interactions have occurred. At this time, all agents override their current leader state from the slow protocol with that of the fast protocol. When the outer phase clock ticks (i.e., it reaches $m$ ), at least one leader exists. We equip each agent $v$ with an additional flag leaderDone ${ }_{v}$, initially set to FALSE. It is set to TRUE as soon as the outer phase clock ticks.

Lemma 6 ([18]). The uniform protocol LEADER_ELECT elects a unique leader. It stabilizes in $\mathrm{O}\left(n \log ^{2} n\right)$ interactions, using $\mathrm{O}(\log \log n)$ many states, w.h.p. Furthermore, after at most $\mathrm{O}\left(n \log ^{2} n\right)$ interactions all agents $v$ have leaderDone $v$ set to TRUE w.h.p., and at that time there is exactly one leader w.h.p.

Fast Leader Election. In [8] the authors describe a stable leader election protocol that admits a trade-off between the running time and the number of states. When using Õ $(n)$ states, their protocol stabilizes w.h.p. in $\mathrm{O}(n \log n)$ interactions. In the following, we call this protocol FASTLEADERElection. The main idea of the protocol is to use $\mathrm{O}(\log n)$ random bits to reduce the number of active leaders much faster than in the original protocol from [18].

Lemma 7 ([8]). The uniform protocol FASTLEADERELECTION elects a unique leader. It stabilizes in $\mathrm{O}(n \log n)$ interactions, using $\tilde{\mathrm{O}}(n)$ many states, w.h.p. Furthermore, after at most $\mathrm{O}\left(n \log ^{2} n\right)$ interactions all agents have leaderDone ${ }_{u}$ set to TRUE w.h.p.

\section{Approximate Counting}

In this section we show Theorem 1. In Section 3.1 we assume that a unique leader exists and we present a protocol which calculates $\lfloor\log n\rfloor$ or $\lceil\log n\rceil$ using a load balancing algorithm. In Section 3.2 we analyze the protocol. In Section 3.3 we show how to combine the protocol from Section 3.1 with a leader election protocol. This shows the first part of Theorem 1. Finally, in Section 3.4 we show how to build a stable protocol by showing the correctness of our error detection mechanism. This shows the second and the third part of Theorem 1. 


\subsection{Approximating $n$ with a Leader}

In this section we assume that a unique leader is given and that all agents are synchronized via the phase clocks. The main idea of our algorithm is to inject an increasing amount of tokens into the system and to use a load balancing routine to estimate the number of agents in the system. The process is finished as soon as roughly $n$ tokens are injected into the system. To save on the number of states, the agents do not store the exact number of tokens, they hold but the logarithm of that number.

Every agent $v$ stores two variables $\left(k_{v}\right.$, searchDone $\left._{v}\right) \in\{-1,0,2, \ldots\} \times\{0,1\}$, initially set to $(-1,0)$. The variable $k_{v}$ stores the logarithm of the load of agent $v$, where the special value

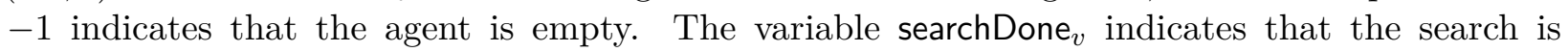
finished. The leader $u$ now orchestrates a linear search over $k_{u} \in\{0,1, \ldots\}$ and finds $k_{u}^{*}$ for which $\log n-1<k_{u}^{*}<\log n+1$. The protocol runs in rounds consisting of multiple phases each. At the beginning of round $r$ the leader injects $2^{r}$ many tokens into the system. The load is then balanced using a powers-of-two load balancing process which restricts the load of any agent to a power of two. The search stops once an agent $v$ has load larger than $1\left(k_{v}>0\right)$. At this time the leader $u$ sets searchDone $_{u}$ to TRUE and broadcasts the value to all agents using one-way epidemics.

In the classical load balancing process (cf. [10]), it is assumed that $m$ indistinguishable tokens are distributed arbitrarily among $n$ agents. At interaction $t$, the load vector $\mathbf{L}(t)$ is defined as $\mathbf{L}(t)=\left(\ell_{1}(t), \ldots, \ell_{n}(t)\right) \in \mathbb{N}_{0}^{n}$, where $\ell_{i}(t)$ is the number of tokens (load) present at agent $i$ at that interaction. Assume agents $u$ and $v$ balance their loads in interaction $t \geq 1$, then $\left(\ell_{u}(t+1), \ell_{v}(t+1)\right)=\left(\left\lfloor\left(\ell_{u}(t)+\ell_{v}(t)\right) / 2\right\rfloor,\left\lceil\left(\ell_{u}(t)+\ell_{v}\right) / 2\right\rceil\right)$. For the powers-of-two load balancing process we define the logarithmic load vector $\mathbf{K}=\left(k_{1}, \ldots, k_{n}\right) \in\{-1,0,1, \ldots\}^{n}$ such that for any agent $v$ we have $\ell_{v}(t)=2^{k_{v}}$ if $k_{v} \geq 0$ and $\ell_{v}(t)=0$ if $k_{v}=-1$. Assume now that agent $u$ interacts with agent $v$. A balancing action is only permitted if either $u$ or $v$ is empty (i.e., $k_{u}=-1$ or $\left.k_{v}=-1\right)$ and both of them are not the leader. Let $k_{u}^{\prime}$ and $k_{v}^{\prime}$ be the resulting logarithmic load values. Then

$$
\left(k_{u}^{\prime}, k_{v}^{\prime}\right)= \begin{cases}\left(k_{u}-1, k_{u}-1\right) & \text { if } k_{u}>0 \text { and } k_{v}=-1 \\ \left(k_{v}-1, k_{v}-1\right) & \text { if } k_{u}=-1 \text { and } k_{v}>0 \\ \left(k_{u}, k_{v}\right) & \text { otherwise. }\end{cases}
$$

The SEARCH Protocol is defined in Algorithm 1. The protocol runs in rounds of 5 phases. Every agent $v$ uses the variable phase ${ }_{v}$ to count the number of phases modulo 5 and the flag firstTick which is set to TRUE when $v$ initiates the first interaction of a phase (see Section 2). In Phase 1 and Phase 4 the leader is active, in the remaining phases the non-leaders are active.

- Phase 0 and Phase 1 are used for the initialization. Every agent $v$ which is not the leader resets $k_{v}$ to -1 (Line 11). During the first interaction $(u, v)$ in Phase 1 the leader $u$ transfers $2^{k_{u}}$ tokens to $v$ (Line 3 ).

- In Phase 2, the non-leader agents perform powers-of-two load balancing, as described above. (Line 14). Phase 3 consists of one-way epidemics where the agents communicate their highest load value (Line 16).

- In Phase 4 (Line 5), the leader $u$ decides if the search is finished or not. If the maximum logarithmic load is less than 1 it concludes that the injected load was smaller than or equal to $\log n-1$. The leader $u$ therefore proceeds to the next round and injects twice as many tokens. If the observed maximum logarithmic load is larger than 1, the leader concludes the protocol by setting searchDone ${ }_{u}$ to TRUE. 


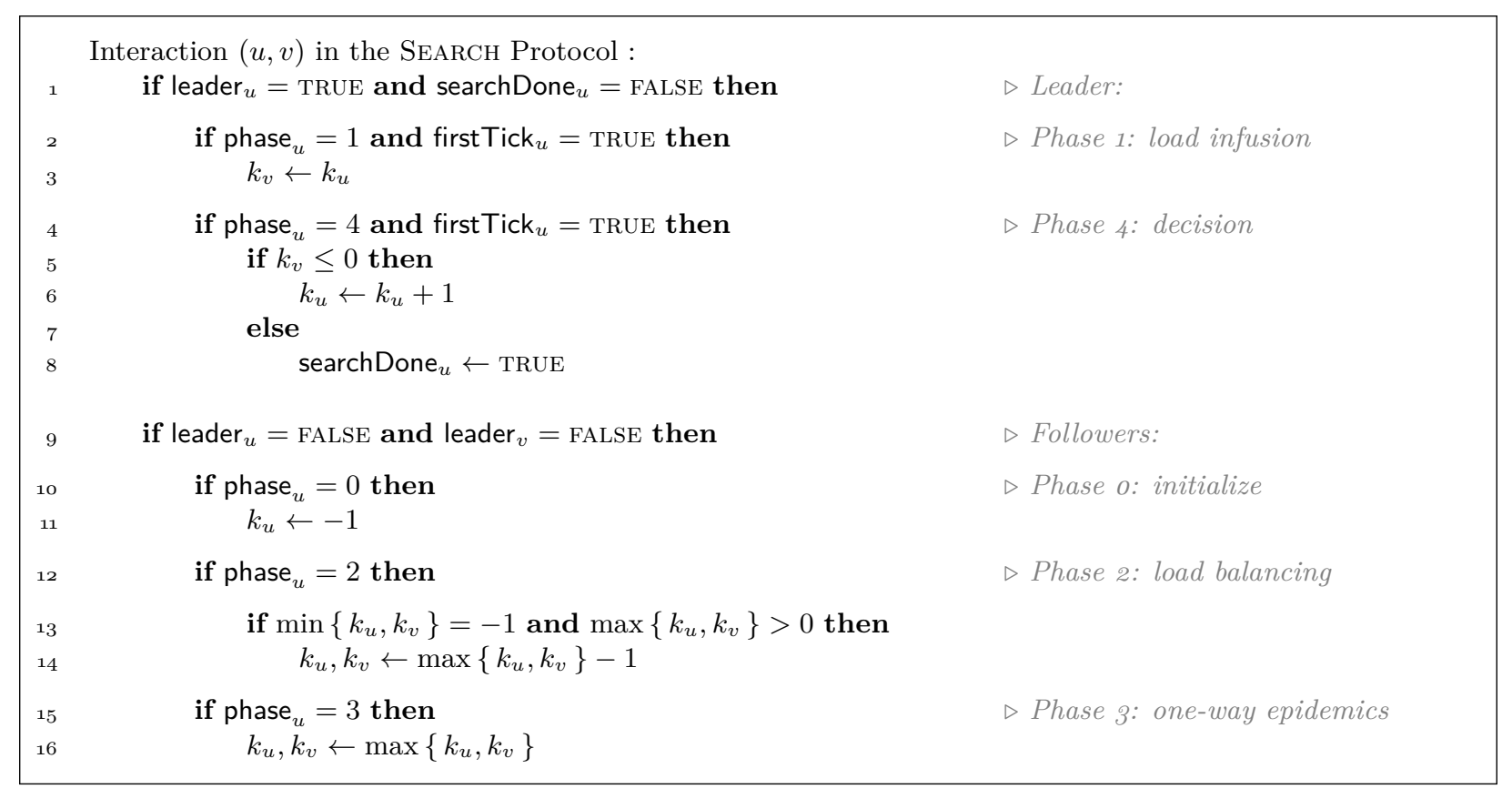

Algorithm 1: The Search Protocol, centerpiece of protocol Approximate

The number of agents is now estimated as $2^{k_{u}}$. In the following, the state of an agent $v$ in this algorithm is called $s(v)$. The state space $S$ is the Cartesian product of the domains of the individual variables ( phase $_{v}$, phase $_{v}$, firstTick , leader $_{v}, k_{v}$, searchDone ${ }_{v}$ ). For an overview over the states of node $v$ in the SEARCH Protocol, see Figure 1.

$$
\begin{aligned}
& s(v)=(\underbrace{\text { phase }_{v}, \text { firstTick }_{v}}_{\text {Phase Clocks }}, \underbrace{\text { leader }_{v}}_{\text {Leader Election }}, \underbrace{k_{v}, \text { searchDone }_{v}}_{\text {SEARCH Protocol }}) \\
& S=\{0, \ldots, 4\} \times\{0,1\} \times\{0,1\} \times\{-1,0,1, \ldots\} \times\{0,1\}
\end{aligned}
$$

Figure 1: State space of the SEARch Protocol (Algorithm 1)

\subsection{Analysis of the SEArch Protocol}

Based on the definition in Equation (1) we first show that the powers-of-two load balancing process on $n$ agents balances the load such that the maximum load is at most 1 (i.e., $k_{v} \leq 0$ for all agents $v$ ) as long as at most $3 / 4 n$ tokens are injected by the leader.

Lemma 8. Assume there exists an agent $u$ with $k_{u}(0)=\kappa$ and $k_{v}(0)=-1$ for all $v \neq u$. If $2^{\kappa} \leq 3 / 4 \cdot n$, then w.h.p. after $t=16 n \log n$ interactions $\max _{v}\left\{k_{v}(t)\right\}=0$.

The proof of Lemma 8 uses the same ideas and shows a similar statement as in Lemmas 2 and 3 from [10] for the classical load balancing process.

In the following, we assume that precisely one agent $u$ is the leader and all agents synchronize themselves via the phase clocks. Based on this assumption we can show the following lemma for the SEARCH Protocol.

Lemma 9. After at most $\mathrm{O}(\log n)$ rounds the leader $u$ sets searchDone ${ }_{u}$ to TRUE, w.h.p. At that time we have w.h.p. that $3 / 4 \cdot n<2^{k_{u}} \leq 2^{\lceil\log n\rceil}$. 


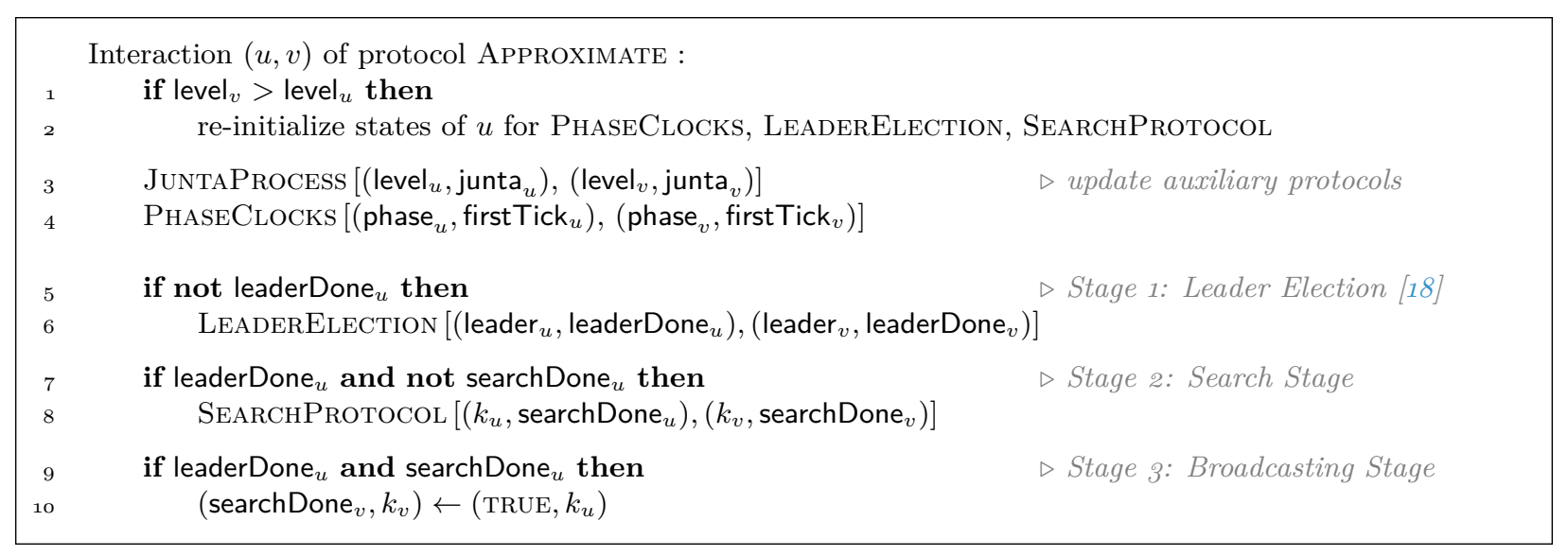

Algorithm 2: Protocol Approximate

Figure 2: State space of protocol Approximate (Algorithm 2)

Proof. The SEArch Protocol runs in multiple phases. In the following, we combine every five consecutive phases to a round such that round $r \geq 0$ consists of phases $5 r$ to $5 r+4$. For a fixed round $r$ let $k_{v}(r)$ be the value of variable $k$ of agent $v$ at the beginning of round $r$. Assuming that all agents are properly synchronized by the phase clocks (see Lemma 5), we can w.h.p. assume that the number of interactions in any round is sufficient for spreading information with one-way epidemics (see Lemma 3) and for the powers-of-two load balancing (see Lemma 8).

Observe that the leader sets searchDone ${ }_{u}$ to TRUE in Line 8 of Phase 4 of the SEARCH Protocol. Let $v$ be an agent with maximal load at the beginning of Phase 3 . In Phase 3 the agents used one-way epidemics to disseminate the value $k_{v}$ to all agents. Hence, searchDone ${ }_{u}$ is set to TRUE in the first round $r$ where an agent $v$ has $k_{v}(r)>0$ after the load balancing, meaning agent $v$ has a load of at least two.

To show the lemma we consider 3 cases, depending on the value $k_{u}(r)$ which corresponds to the load injected by the leader at the beginning of round $r \geq 0$.

Case $2^{k_{u}(r)} \leq 3 / 4 \cdot n$ : In Phase 1 the leader injects $2^{r}$ many tokens. According to Lemma 8, w.h.p. at the end of Phase 2 of Round $r$ no agent will have a load larger that one, meaning for all agents $v$ we have $k_{v} \leq 0$. The maximum value $k_{v}$ which is communicated via one-way epidemics is at most zero and the leader does not set searchDone ${ }_{u}$ to TRUE in Phase 4 of Round $r$. Hence, it injects $2^{r+1}$ tokens at the beginning of the next round.

Case $3 / 4 \cdot n<2^{k_{u}(r)}<n$ : Again, in Phase 1 the leader injects $2^{r}$ many tokens. In this case either there exists an agent $v$ with a load larger than one $\left(k_{v}>0\right)$ at the end of Phase 2 , or for all agents $v$ we have $k_{v} \leq 0$. In the latter case we are back to the previous case: the leader does not set searchDone $_{u}$ to TRUE in Phase 4 of Round $r$. Hence, it injects $2^{r+1}$ tokens at the beginning of the next round. If there exists an agent $v$ with $k_{v}>0$ the leader sets searchDone ${ }_{u}$ to TRUE. 
Case $n \leq 2^{k_{u}(r)}$ : In Phase 1 the leader injects $2^{r} \geq n$ many tokens. The agents balance at least $n$ tokens on $n-1$ agents (since the leader does not participate in the load balancing). Hence, at the end of Phase 2 of Round $r$ there exists an agent with a load of at least two, meaning $k_{v} \geq 1$. The leader sets searchDone ${ }_{u}$ to TRUE in Phase 4 of Round $r$.

From the above cases it follows that after at $\operatorname{most}\lceil\log n\rceil$ rounds searchDone $e_{u}$ is set to TRUE and then $3 / 4 \cdot n<2^{k_{u}} \leq 2^{\lceil\log n\rceil}$.

\subsection{Combining the Search Protocol with Leader Election}

In this section we combine the SEARCH Protocol with the LEAdERELECTION protocol from [18]. The combined protocol works as follows. All agents run the JunTaProcess and the PhaseClocks protocols in parallel to the LEADERELECTION protocol and, later, the SEARCH Protocol. In the junta process, agents cannot decide whether they have already reached the maximal junta level. Therefore, some agents already perform some interactions of the LEADERELECTION protocol or the SEARCh Protocol at a lower junta level. These agents might be badly synchronized, since the phase clock ticks correctly w.h.p. only on the maximal junta level. We therefore define the following procedure that has already been used in [18]: Whenever an agent $u$ interacts with an agent $v$ in a higher level (level $u<$ level $_{v}$ ) all variables for PhaseClocks, LeAderElection and the SEARCH Protocol are re-initialized. In that way, all agents start the LEADERELECTION protocol and the SEARCH Protocol at the maximal junta level from a clean state.

The protocol uses two flags leaderDone $v$ and searchDone ${ }_{v}$ for each agent $v$, which are initially set to FALSE. The first flag, leaderDone ${ }_{v}$, is set once an agent has concluded the leader election protocol (see Section 2). The second flag, searchDone ${ }_{v}$, is set by the SEARch Protocol (see Section 3.1). The two flags leaderDone ${ }_{v}$ and searchDone ${ }_{v}$ allow agent $v$ to distinguish between three stages of the execution of the protocol, the Leader Election Stage (1), the Search Stage (2), and the Broadcasting Stage (3).

In the Leader Election Stage all agents use the protocol from [18] to elect a leader. Recall that the flag leaderDone ${ }_{u}$ is set to TRUE once the external phase clock ticks (see Section 2). Note that at this time there exists exactly one leader w.h.p. [18]. In the Search Stage, we use the SEARCH Protocol defined in Section 3.1. In the Broadcasting Stage the leader uses one-way epidemics to inform all other agents of its value $k_{u}$.

We now give the proof of the first part of Theorem 1, where we show that protocol ApPROXIMATE computes either $\lfloor\log n\rfloor$ or $\lceil\log n\rceil$ w.h.p.

Proof of Statement 1) of Theorem 1. From Lemma 4 it follows that after $\mathrm{O}(n \log n)$ interactions the junta is elected and all agents are inactive. From that point on no agent in the protocol is ever re-initialized again. Let $u$ be the single leader that according to Lemma 6 w.h.p. concludes the leader election protocol. According to Lemma 9, the leader $u$ sets searchDone ${ }_{u}$ to TRUE after at most $\lceil\log n\rceil$ phases of $\mathrm{O}(n \log n)$ interactions each, and at that time $k_{u}$ is either $\lfloor\log n\rfloor$ or $\lceil\log n\rceil$ w.h.p. According to Lemma 3 , within $\mathrm{O}(n \log n)$ further interactions all agents know the value $k_{u}$ from the leader $u$. Together, this gives a total number of $\mathrm{O}\left(n \log ^{2} n\right)$ interactions, after which all agents know the value $k_{u}$ from the leader and thus output either $\lfloor\log n\rfloor$ or $\lceil\log n\rceil$ w.h.p.

Regarding the required number of states we observe that level $v_{v}$ from the junta process and $k_{v}$ from the SEARCH Protocol are the only variables of an agent $v$ that are not of constant size and thus may grow with the population size $n$. For an overview over the states of agent $v$, see Figure 2 . Note that level $v$ is bounded w.h.p. by $\mathrm{O}(\log \log n)$ Lemma 4 , and $k_{v}$ is bounded according to Lemma 9 w.h.p. by $\mathrm{O}(\log n)$. Together this gives that w.h.p. the state space has size $\mathrm{O}(\log \log n \cdot \log n)$. This concludes the proof of Statement 1) of Theorem 1. 


\subsection{The Stable Protocol}

In this section we sketch our stable protocol and proofs of Statements 2) and 3) of Theorem 1. The details can be found in the appendix. Our stable protocol is a hybrid protocol which combines the protocol Approximate with a slow protocol which always finds the correct solution.

The slow protocol works as follows. Every agent starts with exactly one token. Whenever two agents interact and both have the same amount of tokens, one of them hands its tokens over to the other. The agents do not store the exact number of tokens they hold but the logarithm of that number. Note that, due to the definition of the process, the load of every agent is a power of two. After $\mathrm{O}\left(n^{2} \log n\right)$ many interactions one agent will store $\lfloor\log n\rfloor$ many tokens. For each $0 \leq i<\lfloor\log n\rfloor$ there might be one agent storing $2^{i}$ tokens (depending on the value of $n$ ). The number of states required by this process is $\mathrm{O}(\log n)$ if it is sufficient for all but $\log n$ agents to know the approximation for $n$. Otherwise, the value $\lfloor\log n\rfloor$ has to be sent to every agent via one-way epidemics. In this case there are up to $\log n$ many agents that need $\mathrm{O}\left(\log ^{2} n\right)$ many states (exactly those agents which store a value $2^{i}$ ).

The hybrid protocol now works as follows. It first runs Approximate, then the leader $u$ checks if the approximated value $k_{u}$ is correct by injecting $2^{k}$ tokens and by balancing them. If the value of $k_{u}$ is not correct it switches the output over to the slow protocol. The hybrid protocol stabilizes w.h.p. within $\mathrm{O}\left(n \log ^{2} n\right)$ many interactions using $\mathrm{O}\left(\log \log n \cdot \log ^{2} n\right)$ many states if all agents have to know the approximation of $n$, otherwise $\mathrm{O}(\log \log n \cdot \log n)$ states are sufficient.

\section{Counting the Exact Population Size}

In this section we consider the problem of counting the exact population size and present our protocol CountExact. The main idea is as follows. The protocol first selects a junta using the modified junta process from [8] (see Section 2). It then creates phase clocks and selects a leader using the FAstLeaderElection protocol (see Section 2). Again, every agent $v$ has two flags leader $_{v}$ and leaderDone ${ }_{v}$ which indicate whether $v$ is a leader and $v$ has completed the leader election protocol, respectively. Recall that the junta uses a variable level $v$ that stores the maximum level which is reached during the junta election. In [8] it has been shown that in the modified junta process the level reaches w.h.p. a value of $\log \log n \pm c$ for some constant $c$. Hence, we can use $2^{2^{\text {level }}}$ as a first approximation for $n$. This approximation will be refined in two stages, called ApproximationStage and RefinementStage, which are described in Section 4.1 and Section 4.2, respectively. The protocol CountExACT can be found in Algorithm 3. The output of every agent in the protocol CountExact is defined by the RefinementStage.

\subsection{Fast Approximation}

In this section we assume that a unique leader has been elected and that all agents are synchronized via the phase clocks. Additionally, we assume that all agents are on the same maximal junta level level* w.r.t. the modified junta process from [8].

The goal of protocol ApproximationStage is to compute $\log n$ up to an additive error of \pm 3 . The protocol starts with injecting $2^{2^{\text {level }_{u}-8}}=n^{\eta}$ tokens into the system for some $0<\eta \leq 1$. It alternates between increasing the number of injected tokens by a factor of $n^{\eta}$ and load balancing until the total number of tokens $M$ is at least $n / 2$. Unfortunately it is possible that $M$ is very close to $n^{1+\eta}$, resulting in a multiplicative error. Hence, the protocol outputs $k_{u}=\log M-\left\lfloor\log l_{u}\right\rfloor$. In Lemma 10 we will show that $\log n-3 \leq k_{u} \leq \log n+3$. 
Interaction $(u, v)$ of protocol CounTEXACT :

if level $v>$ level $_{u}$ then MENTSTAGE

re-initialize states of $u$ for PhaseClocks, FastleaderElection, ApproximationStage, Refine-

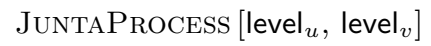

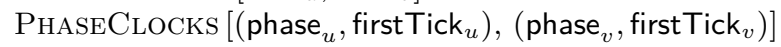

$\triangleright$ update auxiliary protocols

if not leaderDone ${ }_{u}$ then

$\triangleright$ Stage 1: Fast Leader Election

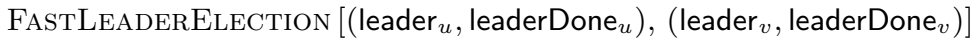

if leaderDone ${ }_{u}$ and not $\mathrm{ApxDone}_{u}$ then $\triangleright$ Stage 2: Approximation Stage ApproximationStage $\left[\left(k_{u}, l_{u}\right.\right.$, ApxDone $\left._{u}\right),\left(k_{v}, l_{v}\right.$, ApxDone $\left.\left._{v}\right)\right]$

if leaderDone ${ }_{u}$ and ApxDone ${ }_{u}$ then RefinementStage $\left[\left(k_{u}, l_{u}\right),\left(k_{v}, l_{v}\right)\right]$

$\triangleright$ Stage 3: Refinement Stage

\section{Algorithm 3: Protocol CountExact}

\begin{tabular}{|c|c|c|c|c|}
\hline state of node $u$ : & $(\underbrace{\text { level }}{ }_{u}$ & $\underbrace{\text { phase }_{u}, \text { firstTick }_{u},}$ & $\underbrace{\text { leader }_{u}}$, leaderDone ${ }_{u}$ & $\underbrace{i_{u}, k_{u}, l_{u}, \text { ApxDone }_{u}})$ \\
\hline state space: & $\begin{array}{l}\text { Junta Process } \\
\qquad \mathbb{N}_{0} \times\end{array}$ & $\begin{array}{l}\text { Phase Clock } \\
\mathbb{N}_{0} \times\{0,1\}\end{array}$ & $\begin{array}{l}\text { Leader Election } \\
\{0,1\} \times\{0,1\}\end{array}$ & $\begin{array}{c}\text { Approximation and Refinement Stages } \\
\times \mathbb{N}_{0} \times\{-1,0,1, \ldots\} \times \mathbb{N}_{0} \times\{0,1\}\end{array}$ \\
\hline
\end{tabular}

Figure 3: State space of protocol CountExACT (Algorithm 3)

Interaction $\left[\left(k_{u}, l_{u}, \mathrm{ApxDone}_{u}\right),\left(k_{v}, l_{v}, \mathrm{ApxDone}_{v}\right)\right]$ in the ApproximationStage protocol:

if firstTick f $_{u}=$ TRUE then

if leader $_{u}=$ TRUE and $i_{u}=0$ then $\quad \triangleright$ initialize first phase

$l_{u} \leftarrow 1$

if leader and $_{u} \geq 4$ then

$\triangleright$ found an approximation

ApxDone $_{u} \leftarrow$ TRUE

$k_{u} \leftarrow i_{u} \cdot 2^{\operatorname{level}_{u}-8}-\left\lfloor\log l_{u}\right\rfloor$

$\left(i_{u}, l_{u}\right) \leftarrow\left(i_{u}+1, l_{u} \cdot 2^{2^{\operatorname{level}_{u}-8}}\right)$

$\triangleright$ start new phase: load explosion

$\left(l_{u}, l_{v}\right) \leftarrow\left(\left\lfloor\left(l_{u}+l_{v}\right) / 2\right\rfloor,\left\lceil\left(l_{u}+l_{v}\right) / 2\right\rceil\right)$

$\triangleright$ classical load balancing

$9 \quad$ ApxDone $_{u} \leftarrow \max \left\{\right.$ ApxDone $_{u}$, ApxDone $\left._{v}\right\}$

$\triangleright$ broadcast ApxDone

\section{Algorithm 4: CountExaCt Approximation Stage}

Interaction $\left[\left(k_{u}, l_{u}\right),\left(k_{v}, l_{v}\right)\right]$ in the RefinementStage protocol:

if phase ${ }_{u}=0$ then

$\triangleright$ initialize agents and broadcast $k_{u}$

$\left(k_{u}, k_{v}, l_{u}, l_{v}\right) \leftarrow\left(\max \left\{k_{u}, k_{v}\right\}, \max \left\{k_{u}, k_{v}\right\}, 0,0\right)$

if firstTick ${ }_{u}=$ TRUE then

if phase $_{u}=1$ and leader ${ }_{u}=$ TRUE then

$\triangleright$ the leader starts with load $2^{8} \cdot 2^{k_{u}}$

$l_{u} \leftarrow 2^{8} \cdot 2^{k_{u}}$

$\triangleright$ multiply load with $2^{k_{u}}$

if phase ${ }_{u}=2$ then

$l_{u} \leftarrow l_{u} \cdot 2^{k_{u}}$

$8 \quad\left(l_{u}, l_{v}\right) \leftarrow\left(\left\lfloor\left(l_{u}+l_{v}\right) / 2\right\rfloor,\left\lceil\left(l_{u}+l_{v}\right) / 2\right\rceil\right)$

Algorithm 5: CountExact Refinement Stage 
The interactions of the protocol are defined in Algorithm 4. The protocol runs in multiple phases of $\mathrm{O}(n \log n)$ interactions each (the phases are determined by the phase clock). Every agent $v$ uses the variables $i_{v}$ (initialized to 0 ) as a phase counter and $l_{v}$ for load balancing. The leader $u$ has an additional variable $k_{u}$ in which it eventually stores the approximation. In the first phase, every agent $v$ initializes its load $l_{v}$ to 0 (non-leaders) or to 1 (leader). In the first interaction of every phase, every agent $v$ increases the phase counter $i_{v}$ and multiplies its load with $n^{\eta}$ (see Line 7 ). During the remainder of the phase, all agents us the classical load balancing process from [10] to balance their tokens. The leader $u$ additionally checks before the multiplication whether it has a load of at least 4 (in which case the total load is at least $2 n$ w.h.p.). If this is the case, the leader calculates the approximation $k_{u}$ as $i_{u} \cdot \eta-\left\lfloor\log l_{u}\right\rfloor$ and raises the flag ApxDone ${ }_{u}$, indicating that the approximation stage has concluded (see Line 5 ). The flag is then sent to all other agents via one-way epidemics (see Line 9), and the raised flag terminates the process.

Lemma 10. Let $u$ be the leader. After at most $\mathrm{O}(n \log n)$ interactions of the Approximation Stage all agents $v$ set ApxDone ${ }_{v}$ to TRUE w.h.p. At that time, $k_{u}=\log n \pm 3$ w.h.p. The protocol uses w.h.p. at most $\tilde{\mathrm{O}}(n)$ states.

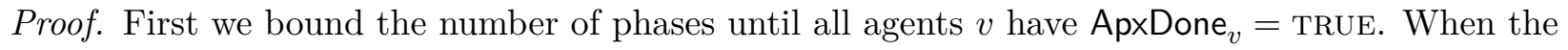
leader $u$ sets ApxDone ${ }_{u}$ to TRUE, all other agents follow via one-way epidemics in the same phase, w.h.p. Therefore it suffices to bound the number of phases until the leader $u$ sets ApxDone A $_{u}$ to TRUE.

Let $\hat{\imath}$ be the first phase in which the leader has a load of at least 4. From load balancing [10] it follows that the total load $M$ at that time is at least $2 n$. At the beginning of Phase 1 the leader $u$ has $n^{\eta}=2^{2^{\text {level }_{v}-8}}$ tokens and all other agents are empty. In the first interaction of Phase $i>1$ all agents $v$ multiply their load with $n^{\eta}$ (see Line 7). Hence, in Phase $i \leq \hat{\iota}$ the total load is $M=n^{i \eta}$, w.h.p. and in Phase $\hat{\imath}$ we have $2 n \leq n^{\hat{\imath} \eta} \leq 6 \cdot n^{1+\eta}$ (see [10]). From Lemma 4 we obtain that w.h.p. $\log \log n-4 \leq$ level $_{v}$ and therefore $\eta \geq 1 / 2^{12}$ w.h.p. Since $n^{\hat{\imath} \eta} \leq 6 \cdot n^{1+\eta}$ we get $\hat{\iota} \leq 1 / \eta+1+\mathrm{o}(1)$. Hence the number of phases $\hat{\iota}$ until $u$ sets ApxDone $u$ to TRUE is bounded by a constant $\hat{\iota}=\mathrm{O}(1)$.

We now bound the quality of the approximation $k_{u}$. In Phase $\hat{\iota}$, the leader sets its variable $k_{u}$ to $k_{u}=\hat{\iota} \cdot 2^{\text {level }_{u}-8}-\left\lfloor\log l_{u}\right\rfloor$. From load balancing [10] we get $l_{u} \leq 2^{\hat{\imath} \cdot 2^{\text {level }_{u}-8}} / n+1.5$ and therefore

$$
\begin{aligned}
k_{u} & \geq \hat{\imath} \cdot 2^{\text {level }_{u}-8}-\log \left(2^{\hat{\iota} \cdot 2^{\text {level }_{u}-8}} / n+1.5\right) \\
& \geq \hat{\imath} \cdot 2^{\text {level }_{u}-8}-\log \left(2^{\hat{\imath} \cdot 2^{\text {leve }_{u}-8}} / n \cdot(1+1.5 / 2)\right) \\
& =\hat{\iota} \cdot 2^{\text {level }_{u}-8}-\log \left(2^{\hat{\imath} \cdot 2^{\text {leve }_{u}-8}}\right)+\log n-\log 1.75 \\
& \geq \log n-3,
\end{aligned}
$$

where (2) holds since $2^{\hat{\imath} \cdot 2^{\text {level }_{u}-8}} \geq 2 n$. Analogously,

$$
\begin{aligned}
k_{u} & \leq \hat{\imath} \cdot 2^{\text {level }_{u}-8}-\log l_{u}+1 \\
& \leq \hat{\iota} \cdot 2^{\text {level }_{u}-8}-\log \left(2^{\hat{\imath} \cdot 2^{\text {evel }_{u}-8}} / n \cdot(1-1.5 / 2)\right)+1 \\
& \leq \log n+3 .
\end{aligned}
$$

It remains to bound the required number of states. For each agent $v$, the variables $i_{v}$ and $k_{v}$ require w.h.p. at most $\mathrm{O}(\log n)$ many states, and the variable $l_{v}$ stores up to $\mathrm{O}\left(n^{\eta}\right) \leq n$ many tokens. 


\subsection{Refining the Approximation}

As before, we assume that a unique leader has been elected and that all agents are synchronized via the phase clocks. We also assume that the leader holds an approximation of $\log n \pm 3$.

In the Refinement Stage, every agent $v$ holds a variables $k_{v}$ for the approximation of $\log n$ and a variable $l_{v}$ that is used for load balancing. The protocol consists of 3 phases. The first phase is used for initialization. The value $k_{u}$ (the approximation calculated by the leader in the previous stage) is spread among all agents via one-way epidemics (Line 2). In the beginning of the second phase the leader injects $2^{8} \cdot 2^{k_{u}}$ many tokens into the system which are balanced, as before. In the beginning of third phase every agent multiplies its load with $2^{k_{u}}$, and then the load is balanced again. In Lemma 11 we show that total number of tokens $M$ is bounded by $2^{2} \cdot n^{2} \leq M \leq 2^{14} \cdot n^{2}$. At the end of the phase every agent can compute $n$ as its output function $\omega(v)=\left\lfloor 2^{8} \cdot 2^{2 k_{v}} / l_{v}\right\rceil$.

Lemma 11. After at most $\mathrm{O}(n \log n)$ interactions of the Refinement Stage, all agents $v$ output $\omega(v)=n$ w.h.p. The protocol uses w.h.p. at most $\tilde{\mathrm{O}}(n)$ states.

Proof. At the end of Phase 0, w.h.p. all agents know $k_{u}$ from the leader according to one-way epidemics. At the end of Phase 1, w.h.p. $C \cdot 2^{k_{u}}$ tokens have been balanced such that $l_{v}=\Theta(1)$ for any agent $v$. At the end of Phase 2 , a total of at least $M \geq 4 \cdot n^{2}$ tokens have been balanced and every agent $v$ has load $l_{v}=\left\lfloor C \cdot 2^{2 k_{u}} / n\right\rceil \pm 1$ w.h.p.

We now need to show that every agent $v$ can output $n$ using its output function $\omega(v)=\left\lfloor C 2^{2 k_{v}} / l_{v}\right\rceil$, provided that $v$ knows the value $k_{v}=k_{u}=\log n \pm 3$ from the leader and the total load $M$ is at least $M=C \cdot 2^{2 k_{u}} \geq 4 n^{2}$ tokens. The proof is based on the proof of Lemma 3.8 from [15].

Observe that after the load balancing, w.h.p. $l_{v}=C \cdot 2^{2 k_{u}} / n+r$ where $r \in[-1.5,1.5]$ (the error term $r$ covers the remaining discrepancy and the rounding error). Let $\hat{\omega}(v)$ be the output of agent $v$ before the rounding is applied. We derive, analogously to [15],

$$
\begin{aligned}
\hat{\omega}(v) & =\frac{M}{l_{v}}=\frac{C \cdot 2^{2 k_{u}}}{C \cdot 2^{2 k_{u}} / n+r}=\frac{C \cdot 2^{2 k_{u}} \cdot n}{C \cdot 2^{2 k_{u}}+r n} \\
& =\frac{C \cdot 2^{2 k_{u}} \cdot n}{C \cdot 2^{2 k_{u}}\left(1+\frac{r n}{C \cdot 2^{2 k_{u}}}\right)}=\frac{n}{1+\frac{r n}{C \cdot 2^{2 k_{u}}}}
\end{aligned}
$$

which gives us, since $r \in[-1.5,1.5]$

$$
\begin{aligned}
\hat{\omega}(v) & \geq \frac{n}{1+\frac{1.5 n}{C \cdot 2^{2 k_{u}}}} \stackrel{\left(M \geq 4 n^{2}\right)}{\geq} \frac{n}{1+\frac{1.5 n}{4 n^{2}}} \\
& =\frac{n}{1+\frac{1}{3 n}}=n-\left(\frac{1}{3}-\frac{1}{3(3 n+1)}\right)>n-\frac{1}{2} \\
\hat{\omega}(v) & \leq \frac{n}{1-\frac{1.5 n}{C \cdot 2^{2 k_{u}}}} \stackrel{\left(M \geq 4 n^{2}\right)}{\leq} \frac{n}{1-\frac{1.5 n}{4 n^{2}}} \\
& =\frac{n}{1-\frac{1}{3 n}}=n+\frac{1}{3}+\frac{1}{3(3 n-1)}<n+\frac{1}{2} .
\end{aligned}
$$

and

Therefore, $\omega(v)=\lfloor\hat{\omega}(v)\rceil=n$.

\subsection{Analysis of CountExact}

We now show Theorem 2. Due to space limitations, we only show that CounTExACT calculates the value of $n$ w.h.p. In the appendix we describe how to combine the protocol with a slow always-correct protocol, which shows that protocol COUnTExACT stabilizes. 
Proof. From Lemma 4 it follows that after $\mathrm{O}(n \log n)$ interactions the junta is elected and all agents are inactive. From that point on no agent in the protocol is ever re-initialized again. Let $u$ be the single leader that w.h.p. concludes the FASTLEADERELECTION protocol according to Lemma 7 after at most $\mathrm{O}(n \log n)$ interactions. According to Lemma 10, the leader $u$ computes $\log n \pm 3$ and

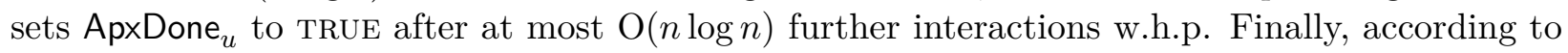
Lemma 11, all agents output $n$ after $\mathrm{O}(n \log n)$ further interactions. The total number of interactions therefore is $\mathrm{O}(n \log n)$. Observe that all protocols require at most $\tilde{\mathrm{O}}(n)$ states. This concludes the proof.

In order to argue that CountExACT stabilizes, we use the same idea as in Section 3.4, where we combined the protocol for approximate counting with a slow protocol which is always correct. Together with an error detection mechanism, this gives a protocol that stabilizes w.h.p. in $\mathrm{O}(n \log n)$ interactions such that every agent outputs the exact value $n$, using $\tilde{\mathrm{O}}(n)$ states. Further details can be found in the appendix.

\section{References}

[1] Dan Alistarh, James Aspnes, David Eisenstat, Rati Gelashvili, and Ronald L. Rivest. Time-space trade-offs in population protocols. In Philip N. Klein, editor, Proceedings of the Twenty-Eighth Annual ACM-SIAM Symposium on Discrete Algorithms, SODA 201\%, Barcelona, Spain, Hotel Porta Fira, January 16-19, pages 2560-2579. SIAM, 2017.

[2] Dan Alistarh, James Aspnes, and Rati Gelashvili. Space-optimal majority in population protocols. In Artur Czumaj, editor, Proceedings of the Twenty-Ninth Annual ACM-SIAM Symposium on Discrete Algorithms, SODA 2018, New Orleans, LA, USA, January 7-10, 2018, pages 2221-2239. SIAM, 2018.

[3] Dan Alistarh, Rati Gelashvili, and Milan Vojnovic. Fast and exact majority in population protocols. In Chryssis Georgiou and Paul G. Spirakis, editors, Proceedings of the 2015 ACM Symposium on Principles of Distributed Computing, PODC 2015, Donostia-San Sebastián, Spain, July 21 - 23, 2015, pages 47-56. ACM, 2015.

[4] Dana Angluin, James Aspnes, Zoë Diamadi, Michael J. Fischer, and René Peralta. Computation in networks of passively mobile finite-state sensors. In Soma Chaudhuri and Shay Kutten, editors, Proceedings of the Twenty-Third Annual ACM Symposium on Principles of Distributed Computing, PODC 2004, St. John's, Newfoundland, Canada, July 25-28, 2004, pages 290-299. ACM, 2004.

[5] Dana Angluin, James Aspnes, Zoë Diamadi, Michael J. Fischer, and René Peralta. Computation in networks of passively mobile finite-state sensors. Distributed Computing, 18(4):235-253, 2006.

[6] Dana Angluin, James Aspnes, and David Eisenstat. Fast computation by population protocols with a leader. Distributed Computing, 21(3):183-199, 2008.

[7] Dana Angluin, James Aspnes, David Eisenstat, and Eric Ruppert. The computational power of population protocols. Distributed Computing, 20(4):279-304, 2007.

[8] Petra Berenbrink, Robert Elsässer, Tom Friedetzky, Dominik Kaaser, Peter Kling, and Tomasz Radzik. Majority \& stabilization in population protocols. CoRR, abs/1805.04586, 2018. 
[9] Petra Berenbrink, Robert Elsässer, Tom Friedetzky, Dominik Kaaser, Peter Kling, and Tomasz Radzik. A population protocol for exact majority with $\mathrm{o}\left(\log ^{5 / 3} n\right)$ stabilization time and $\theta(\log n)$ states. In Ulrich Schmid and Josef Widder, editors, 32nd International Symposium on Distributed Computing, DISC 2018, New Orleans, LA, USA, October 15-19, 2018, volume 121 of LIPIcs, pages 10:1-10:18. Schloss Dagstuhl - Leibniz-Zentrum fuer Informatik, 2018.

[10] Petra Berenbrink, Tom Friedetzky, Dominik Kaaser, and Peter Kling. Tight \& simple load balancing. In Proceedings of the 33rd IEEE International Parallel and Distributed Processing Symposium (IPDPS), Rio de Janeiro, Brazil, 20-24 May 2019, pages 718-726, 2019.

[11] Petra Berenbrink, Dominik Kaaser, Peter Kling, and Lena Otterbach. Simple and efficient leader election. In 1st Symposium on Simplicity in Algorithms, SOSA 2018, January 7-10, 2018, New Orleans, LA, USA, pages 9:1-9:11, 2018.

[12] Andreas Bilke, Colin Cooper, Robert Elsässer, and Tomasz Radzik. Brief announcement: Population protocols for leader election and exact majority with $O\left(\log ^{2} n\right)$ states and $O\left(\log ^{2} n\right)$ convergence time. In Elad Michael Schiller and Alexander A. Schwarzmann, editors, Proceedings of the ACM Symposium on Principles of Distributed Computing, PODC 201\%, Washington, DC, USA, July 25-27, 201\%, pages 451-453. ACM, 2017.

[13] David Doty and Mahsa Eftekhari. Efficient size estimation and impossibility of termination in uniform dense population protocols. CoRR, abs/1808.08913, 2018.

[14] David Doty, Mahsa Eftekhari, Othon Michail, Paul G. Spirakis, and Michail Theofilatos. Brief announcement: Exact size counting in uniform population protocols in nearly logarithmic time. In 32nd International Symposium on Distributed Computing, DISC 2018, New Orleans, LA, USA, October 15-19, 2018, pages 46:1-46:3, 2018.

[15] David Doty, Mahsa Eftekhari, Othon Michail, Paul G. Spirakis, and Michail Theofilatos. Exact size counting in uniform population protocols in nearly logarithmic time. CoRR, abs/1805.04832, 2018.

[16] David Doty and David Soloveichik. Stable leader election in population protocols requires linear time. Distributed Computing, 31(4):257-271, 2018.

[17] Moez Draief and Milan Vojnovic. Convergence speed of binary interval consensus. SIAM J. Control and Optimization, 50(3):1087-1109, 2012.

[18] Leszek Gasieniec and Grzegorz Stachowiak. Fast space optimal leader election in population protocols. In Proceedings of the Twenty-Ninth Annual ACM-SIAM Symposium on Discrete Algorithms, SODA 2018, New Orleans, LA, USA, January 7-10, 2018, pages 2653-2667. SIAM, 2018.

[19] Adrian Kosowski and Przemyslaw Uznanski. Brief announcement: Population protocols are fast. In Proceedings of the 2018 ACM Symposium on Principles of Distributed Computing, PODC 2018, Egham, United Kingdom, July 23-27, 2018, pages 475-477, 2018.

[20] George B. Mertzios, Sotiris E. Nikoletseas, Christoforos L. Raptopoulos, and Paul G. Spirakis. Determining majority in networks with local interactions and very small local memory. Distributed Computing, 30(1):1-16, 2017. 


\section{A. Load Balancing}

In this appendix we show Lemma 8. The proof follows along the lines of the proofs of Lemmas 2 and 3 from [10]. We re-state the lemma as follows.

Lemma 8. Assume there exists an agent $u$ with $k_{u}(0)=\kappa$ and $k_{v}(0)=-1$ for all $v \neq u$. If $2^{\kappa} \leq 3 / 4 \cdot n$, then w.h.p. after $t=16 n \log n$ interactions $\max _{v}\left\{k_{v}(t)\right\}=0$.

Proof. In the following, we will argue about individual tokens. However, this is only used for the sake of the analysis. In the actual process, all agents store at all times only the logarithm of their current numbers of tokens. We assume that each agent $u$ holds a number of $l_{u}(t)=2^{k_{u}(t)}$ tokens which are stored in some order. Initially, the tokens are ordered arbitrarily. We define the height $h(b)$ of a token $b$ as the number of tokens below $b$ w.r.t. that order. Whenever an agent $u$ interacts with another agent $v$ that does not have any load, every second token is moved from agent $u$ to agent $v$ and stored on $v$ in the original order. See Figure 4 for an example. Therefore, all tokens

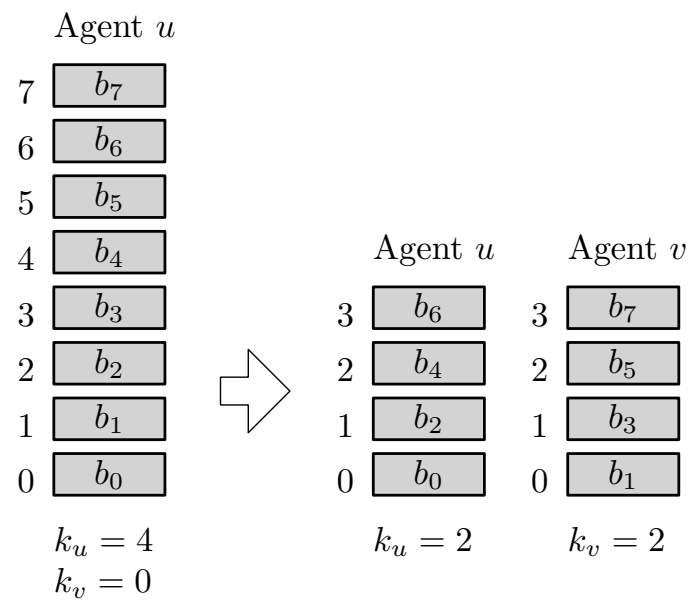

Figure 4: Example: Agent $u$ moves every second token to the previously empty agent $v$.

participating in the interaction reduce their height by a factor of at least 2 . According to the assumptions of Lemma 8 we have $2^{\kappa} \leq 3 / 4 \cdot n$ and thus there are at most $3 / 4 \cdot n$ tokens in the system. Even when spread out as much as possible, these tokens cannot take up more than $3 / 4 \cdot n$ agents. Therefore, the probability that a specific token $b$ of height $h(b)>0$ reduces its height by a factor of 2 when participating in an interaction with an empty agent is at least $1 / 4$. (Note that the actual probability is bounded only by $1 / 4 \cdot(1-1 / n)$, since the leader does not participate in the load balancing process. For simplicity we work with the expression 1/4, and for $n$ large enough our final results holds nonetheless.) We now observe that

1) the agent on which $b$ is stored is selected with probability $2 / n$, and

2) the other agent selected does not have any load with probability $1 / 4$.

Combining these two events gives us that in interaction $t$ with probability $1 /(2 n)$ the token $b$ reduces its height by at least a constant factor of 2 or has already reached height 0 . We denote this event as a good interaction $t$ for token $b$. We now consider $16 n \log n$ interactions and use $X_{b, i}$ to denote an indicator random variable for good interactions of token $b$. Since for one specific token the interactions occur independently from each other, we may apply Chernoff bounds to 
$X_{b}=\sum_{i=1}^{16 n \log n} X_{b, i}$ and obtain

$$
\operatorname{Pr}\left[X_{b}<\log n-1\right]<\exp \left(-\frac{49}{16} \log n\right)<n^{-3} .
$$

From the union bound over all tokens we obtain that after at most $16 n \log n$ interactions all tokens have reached height 0 with probability $1-n^{-2}$.

\section{B. The Stable Protocol}

In this appendix we show that we can build a stable protocol using only an additional multiplicative factor of $\mathrm{O}(\log n)$ states. We first describe our error detection mechanisms and then show Statement 2) of Theorem 1. Finally, we show how to reduce the required number of states by accepting a small number of agents that do not output the correct result.

Our approach is closely related to the idea of building a hybrid protocol in [18] (see also Section 2). We need to handle two possible sources of errors. The first type of error comes from leader election, where it may happen that no agent ever completes the fast leader election protocol, or multiple leaders do. The second type of error occurs when the SEARCH Protocol fails.

If no agent completes leader election, then no agent will ever set leaderDone. In that case, we use the following solution: From the very beginning, all agents run a slow backup protocol in parallel. (We give a description of the backup protocol in Appendix C.) Only if an agent $v$ completes the leader election protocol and sets leaderDone ${ }_{v}$ to TRUE, it stops the execution of the slow backup protocol and proceeds with the SEARCH Protocol, which is governed by the leader.

If multiple agents conclude the leader election stage as a leader, then this error can be detected whenever two leaders interact with each other. The agent $v$ which detected the error raises an error flag error $v$. This flag is adopted by all other agents using one-way epidemics. When an agent sets its error flag, it ignores all of its previous computations and executes a new instance of the backup protocol. Note that stopping the backup protocol while running the SEARCH Protocol allows us to save states by re-using the states from the backup protocol (at costs of having to re-start the backup protocol in case of a later error).

The second type of error occurs when some agents fail to properly execute the SEARCH Protocol. E.g., either load balancing or maximum broadcast could fail, resulting in an incorrect value $k_{u}$ at the leader $u$. In the remainder of this section, we describe how such an error can be detected after the execution of the SEARCH Protocol. As before, whenever an agent detects an error, it raises the error flag and switches to a new instance of the backup protocol.

To detect an error during the execution of the SEARCH Protocol, all agents use after the SEARCH Protocol (i.e., when ApxDone ${ }_{v}=$ TRUE for agent $v$ ) an additional error detection protocol ERRORDETECTION defined in Algorithm 7. This protocol is enabled by replacing the Broadcasting Stage from the Approximate (Algorithm 2) with the Error Detection Stage by calling the ErrorDetection protocol (Algorithm 7) as follows:

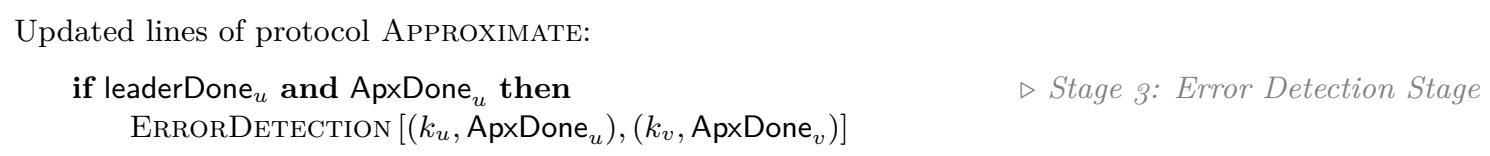

Algorithm 6: Additional Lines for Protocol Approximate to enable error detection 
The error detection protocol runs in 5 phases. We assume that all agents start counting the phases over from 0 when they enter the Error Detection Stage, and they stop counting phases when they have reached phase 4 . The protocol requires at each agent $v$ in addition to the states from Algorithm 1 a flag error $v$ which is initially set to FALSE and an additional variable $l_{v} \in\{0,1, \ldots, 32\}$ used for load balancing. Intuitively, the leader $u$ balances $2^{k_{u}}$ tokens among the agents, and the resulting load at each agent allows the leader to validate its result. The phases of the ERrorDetection protocol have the following purposes.

1) In the first phase ( phase $_{u}=0$ ), the leader $u$ initializes another agent in its first interaction with a load of $2^{k_{u}-2}$ tokens stored in powers of two (Line 4 ). Note that the -2 in the exponent is required that the powers-of-two load balancing process perfectly balances all tokens w.h.p., and it will be compensated in the third phase. All non-leader agents set their load to -1 once they enter the Error Detection stage (Line 2).

2) In the second phase, all agents $v$ except the leader perform the powers-of-two load balancing procedure on their $k_{v}$ values.

3) In the third phase, every agent $v$ initializes its additional token counter $l_{v}$ either to 0 if $k_{v}=-1$ (the agent did not have any tokens in the powers-of-two load balancing process) or to 32 if $k_{v}=0$ (the agent had one token in the powers-of-two load balancing process). Here, the factor of 32 over-compensates for the smaller number of tokens initially used in the first phase.

4) In the fourth phase, all agents $v$ perform the classical load balancing process from [10] on their $l_{v}$-values.

5) In the last phase, all agents perform the following. Every agent $v$ checks if its remaining load $l_{v}$ is at least 3 . If this is not the case, an error has occurred, the agent raises the error flag error $_{v}$ and moves over to the backup protocol. Furthermore, every agent $v$ checks whether the remaining discrepancy is at most 2 . In addition, once an agent $v$ has reached phase $v_{v}=4$, it stops its phase clock. In that way, it runs through the error detection protocol exactly once and then remains in Phase 4 forever (unless an error is detected).

The output of every agent $v$ is the value $k_{u}$ from the leader $u$, which is set in Line 19 . It is then sent to all agents in Phase 4 via one-way epidemics. 


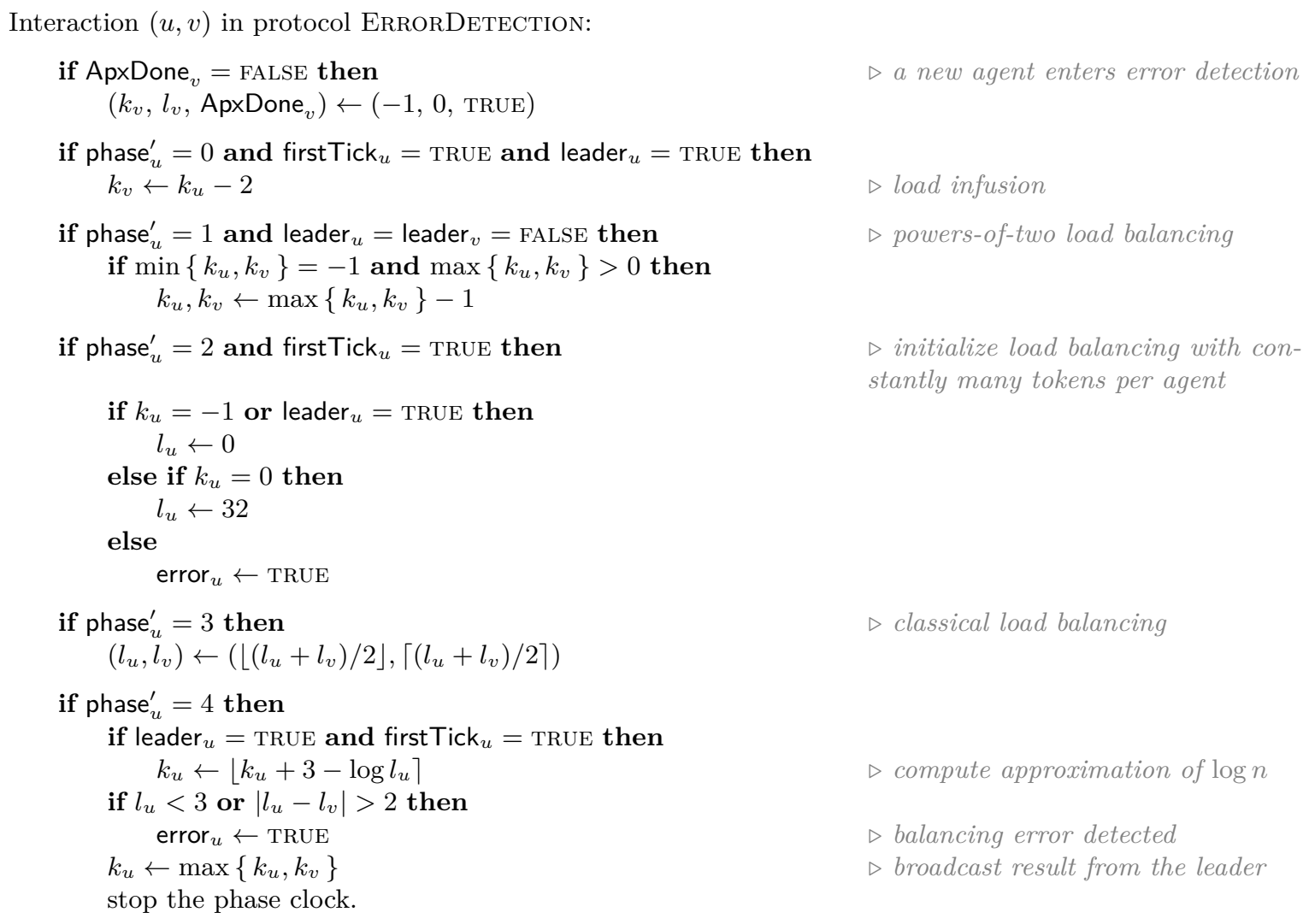

$\triangleright$ a new agent enters error detection

$\triangleright$ load infusion

$\triangleright$ powers-of-two load balancing

$\triangleright$ initialize load balancing with constantly many tokens per agent

$\triangleright$ classical load balancing

$\triangleright$ compute approximation of $\log n$

$\triangleright$ balancing error detected

$\triangleright$ broadcast result from the leader

Algorithm 7: Error Detection protocol in protocol Approximate

We now put everything together and show the stability of Algorithm 2. Recall that in Section 3.2 we have shown that the protocol works as claimed w.h.p. It remains to show that the protocol stabilizes.

Proof of Statement 2) and Statement 3) of Theorem 1. Recall that in any error case, all agents $v$ raise their error $r_{v}$ flag. The error $v$ flag is always adopted by all other agents using one-way epidemics. Whenever an agent $v$ sets its error ${ }_{v}$ flag, it ignores all of its previous computations and starts to execute a new instance of the backup protocol. The backup protocol stabilizes after $\mathrm{O}\left(n \log ^{2} n\right)$ interactions w.h.p. (see Lemma 12) in Appendix C.

We first show that the protocol stabilizes unless an error is detected.

W.h.p., the phase clocks work correctly such that the phase length is sufficiently long enough for load balancing to complete (see Lemma 5). The powers-of-two load balancing process balances then the load in Phase 1 such that all agents $v$ (except the leader $u$ ) have a value $k_{v} \in\{-1,0\}$ (see Lemma 8). The resulting number of tokens is then multiplied with 32 and balanced in Phase 3 using the classical load balancing process from [10].

From the result on load balancing we obtain the following.

- From Lemma 9 we obtain that $k_{u} \geq\lfloor\log n\rfloor$ w.h.p. Therefore, at least $2^{k_{u}-2} \cdot 32>4 \cdot n$ tokens are balanced over $n$ agents. From [10, Theorem 1] we obtain that after balancing every agent has load at least 3 w.h.p. Otherwise, the error flag is raised by every agent which has load less than 3 (see Line 21). 
- As before, the error flag is raised by every agent which observes a balancing error. However, note that after balancing the discrepancy is at most 2 w.h.p. [10]. Therefore, a load balancing error does not occur w.h.p. and thus in Line 21 of Algorithm 7 the error flag remains unset w.h.p.

Once the load balancing has successfully completed such that $l_{v} \geq 3$ at all agents $v$, for all pairs of agents $(u, v)$ we have $\left|l_{u}-l_{v}\right| \leq 2$, all agents are in Phase 4 , and all agents have adopted the maximal value $k_{u}$ from the leader $u$, the protocol does not provide any transitions due to which an agent could change its output. Together this implies that the protocol stabilizes w.h.p. if $k_{u} \in\{\lfloor\log n\rfloor,\lceil\log n\rceil\}$ after the SEARCH Protocol.

Observe that the leader $u$ computes its approximation in the ERRORDETECTION protocol as $k_{u}=\left\lfloor k_{u}+3-\log l_{u}\right\rceil$ (see Line 19). We use $l_{u}=\left\lfloor 32 \cdot 2^{k_{u}}\right\rceil \pm 1$ and get that $k_{u}=\log n \pm \delta$ for some $\delta<1$. Therefore, $k_{u} \in\{\lfloor\log n\rfloor,\lceil\log n\rceil\}$ provided the load balancing has concluded with a maximal remaining discrepancy of 2 .

To now show Statement 2) of Theorem 1 we handle the various sources of errors one after the other.

- Leader Election Errors:

If no leader concludes the leader election protocol from [18], then no agent ever sets leaderDone to TRUE. In that case all agents output the result from the backup protocol indefinitely.

If multiple agents conclude the leader election protocol, then this is detected when two of them interact with each other. In that case, both agents raise the error flag, causing the system to stabilize due to the backup protocol.

- Synchronization Errors:

Observe that the ApxDone flag is transmitted to all agents via one-way epidemics (Line 2 of Algorithm 7). Once the flag is set, the ERRORDETECTION protocol runs in 5 phases counted in the phase' variable. It is crucial that this number of phases is a constant, and that the phase clock gets stuck in Phase 4.

Recall that we define that the phase clocks are updated at the beginning of an interaction. If two agents interact thereafter that have different phase' values, the execution has become asynchronous and the participating agents raise the error flag. Note that this also implies that every agent initiates at least one interaction of every phase (thereby executing the special instructions conditioned on firstTick $=$ TRUE), since otherwise a phase difference of at least 1 is detected in a later interaction.

- Errors in the SEARCH Protocol:

During the SEARCH Protocol, no explicit error detection mechanisms are used. However, observe that as soon as a leader is present the SEARch Protocol, the total load in the system starts to grow over time. This follows from the fact that the phase clock performs an infinite sequence of ticks with probability 1 . Therefore the value $k_{u}$ from the leader is incremented repeatedly, and therefore the total load in the system grows such that the leader will eventually conclude the SEARCH Protocol.

At the time when the leader $u$ concludes the SEARCH Protocol, its estimate $k_{u}$ of $\log n$ might be too small. However, this error is detected in the ERRORDETECTION protocol and the error flag is raised by some agent. As soon as the error flag is raised, all agents adopt the error flag via one-way epidemics, and the protocol then stabilizes via the backup protocol. 
It remains to show that the protocol stabilizes w.h.p. within $\mathrm{O}\left(n \log ^{2} n\right)$ interactions. Recall that without the ERRORDETECTION protocol the protocol converges w.h.p. within $\mathrm{O}\left(n \log ^{2} n\right)$ interactions (see Statement 1) of Theorem 1). The ERRORDETECTION protocol runs in 5 additional phases. Together this gives us that w.h.p. the protocol stabilizes in $\mathrm{O}\left(n \log ^{2} n\right)$ interactions.

Regarding the required number of states, we observe that the ERRORDETECTION protocol operates on the same states as the SEARCH Protocol, and the only non-constant size variable of any agent $u$ is $k_{u}$, which uses w.h.p. up to $\log n$ states - as in the SEARCH Protocol. Except for the Backup Protocol, the stable protocol therefore does not require more states then the SEARCH Protocol. The Backup Protocol requires O $\left(\log ^{2} n\right)$ states (see Appendix C. Since it has to be run in parallel until an agent $u$ sets leaderDone ${ }_{u}$ to TRUE, our protocol requires in total a number of $\mathrm{O}\left(\log ^{2} n \log \log n\right)$ states w.h.p.

It remains to show that we can further reduce the required number of states if we slightly relax the output definition such that only all but $\log n$ agents output the correct result. This is achieved by using a slightly modified backup protocol which requires only $\mathrm{O}(\log n)$ states, but up to $\log n$ agents do not output the correct result.

\section{Backup Protocols}

\section{C.1. Backup Protocol for Approximate Counting}

In this appendix we define our backup protocol to compute $\lfloor\log n\rfloor$, using at most $\log ^{2} n$ states.

In the slow but stable backup protocol, each agent starts with one token. Agents then keep combining tokens in groups of sizes which are powers of two. Each agent $v$ keeps a pair $\left(k_{v}, k_{v}^{\max }\right)$, where $k_{v}$ is the number of tokens in this agent and $k_{v}^{\max }$ is the largest $k_{w}$ that agent $v$ is aware of. Let $k_{v}=-1$ represent an empty agent which does not have any tokens. The initial state of an agent is $(0,0)$, that is, initially each agent has one token. During an interaction of two agents $(u, v)$, there are now two possible cases. If both agents have the same number of tokens, than the first agent takes all of them. Otherwise, if the two agents have different numbers of tokens, no exchange of tokens is possible. In both cases, the agents always update the maximum number of tokens, which is stored in the $k^{\text {max }}$-values. Formally, the protocol has the transitions

$$
\delta\left[\left(k_{u}, k_{u}^{\max }\right),\left(k_{v}, k_{v}^{\max }\right)\right]= \begin{cases}{\left[\left(k_{u}+1, K\right),(-1, K)\right]} & \text { if } k_{u}=k_{v} \geq 0 \\ {\left[\left(k_{u}, K\right),\left(k_{v}, K\right)\right]} & \text { otherwise }\end{cases}
$$

where

$$
K=\max \left\{k_{u}^{\max }, k_{v}^{\max }\right\} \text {. }
$$

We now show the following result for the backup protocol.

Lemma 12. Let $K_{i}=\left\{u: k_{u}=i\right\}$ be the set of agents which have $k_{u}=i$ and let $n_{i}$ be the $i$-th bit of the binary representation of $n$. Then the backup protocol converges to a configuration where

- $\left|K_{i}\right|=n_{i}$ for all $i \geq 0$ and

- $\max _{u}\left\{k_{u}\right\}=\lfloor\log n\rfloor$ and $k_{v}^{\max }=\lfloor\log n\rfloor$ for all agents $v$.

It uses at most $(\log n+1)^{2}$ states and stabilizes w.h.p. within $\mathrm{O}\left(n^{2} \log ^{2} n\right)$ interactions.

The proof follows straightforwardly from the coupon collector's problem. For completeness, we formally show the claim as follows. 
Proof. We show by induction on $j$ in $\{0,1, \ldots,\lfloor\log n\rfloor\}$ that after $j \cdot \mathrm{O}(n \log n)$ interactions for all $i<j$ it holds that $\left|K_{i}\right|=n_{i}$ w.h.p.

The base case trivially holds. For the induction step, fix a $j \in\{0,1, \ldots,\lfloor\log n\rfloor\}$. From the induction hypothesis we obtain that for any level $i<j$ there are $n_{i}$ token stuck on that level. Note that $n_{i} \leq 1$ : this means that no further tokens from any agent in $K_{i}$ with $i<j$ can be merged, and therefore $\left|K_{j}\right|$ cannot increase w.h.p. We now apply a standard coupon collecting argument: After $\mathrm{O}\left(n^{2} \log n\right)$ interactions, as many tokens as possible on level $j$ have been merged w.h.p. Thus, $\left|K_{j}\right|$ is either 0 or 1 after $j \cdot \mathrm{O}(n \log n)$ interactions w.h.p. It remains to argue that $\left|K_{j}\right|=n_{j}$.

From the binary representation of $n$ it follows that the total number of tokens that can ever reach level $j$ is $\hat{k}_{j}=\left(n-\sum_{i=0}^{j-1} n_{j} \cdot 2^{i}\right) / 2^{j}=\left\lfloor n / 2^{j}\right\rfloor$. If $n_{j}=0, \hat{k}_{j}$ is even. All $\hat{k}_{j}$ tokens can therefore be merged to level $j$ such that eventually $\left|K_{j}\right|=0$. If $n_{j}=1$, a single token remains on level $j$ indefinitely. This shows that $\left|K_{j}\right|=n_{j}$ after $j \cdot \mathrm{O}\left(n^{2} \log n\right)$ interactions.

From the binary representation of $n$ it furthermore follows that eventually $\left|K_{\lfloor\log n\rfloor}\right|=1$. Let $u \in K_{\lfloor\log n\rfloor}$ at some time $t$ and observe that $k_{u}=\lfloor\log n\rfloor>k_{v}$ for any $v \neq u$. According to Lemma 3 on one-way epidemics it follows that $k_{u}^{\max }=k_{u}$ is transmitted to all other agents after additional $\mathrm{O}(n \log n)$ interactions after time $t$ w.h.p.

Finally, the protocol requires at each agent at most $\lfloor\log n\rfloor+1$ states for both, the $k_{u}$ and the $k_{u}^{\max }$ values. This concludes the proof.

\section{C.2. Backup Protocol for Exact Counting}

In the backup protocol for exact counting, every agent $u$ has variables $\left(c_{u}, n_{u}\right)$ initially set to (FALSE, 1). Interactions are defined as

$$
\left[\left(c_{u}^{\prime}, n_{u}^{\prime}\right),\left(c_{v}^{\prime}, n_{v}^{\prime}\right)\right]= \begin{cases}{\left[\left(\mathrm{FALSE}, n_{u}+n_{v}\right),\left(\mathrm{TRUE}, n_{u}+n_{v}\right)\right]} & \text { if } c_{u}=c_{v}=\text { FALSE } \\ {\left[\left(c_{u}, \max \left\{n_{u}, n_{v}\right\},\left(c_{v}, \max \left\{n_{u}, n_{v}\right\}\right)\right]\right.} & \text { otherwise }\end{cases}
$$

Intuitively, in this protocol every agent starts with one token and a bit $c_{u}$ indicating whether its token has been counted. Agents combine tokens until eventually a single agent holds all $n$ tokens. Those agents which have already been counted broadcast the maximum value they have observed so far. The following lemma follows directly from coupon collecting.

Lemma 13. The backup protocol defined via Equation (4) stabilizes w.h.p. within $\mathrm{O}\left(n^{2} \log n\right)$ interactions. When it stabilizes, every agent outputs the exact value $n$.

\section{Fast Leader Election using $n^{\varepsilon}$ States}

The following protocol performs leader election in $\mathrm{O}(n \log n)$ interactions using $\mathrm{O}\left(n^{\varepsilon}\right)$ many states for some small constant $\varepsilon>0$. It is a special case of the protocol presented in [8]. It has the

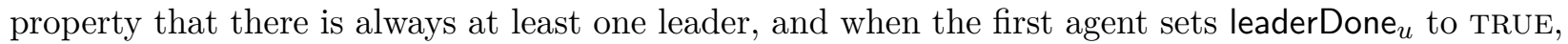
there is at most one leader w.h.p.

To simplify the description of the protocol, we assume that agents can toss a random coin and generate one random bit uniformly and independently in each interaction. While this is not covered by the actual population model, the randomness from the scheduler can be exploited. This technique has been called synthetic coins by Alistarh et al. [1], and a simple analysis has been presented in [11]. Intuitively, the idea is that every agent keeps track of the parity of its current interaction number by flipping a bit in every interaction. This bit then functions as a source of randomness for the interaction partner. 
The FastLeaderEleCtion protocol operates for each agent $u$ on the variables leader ${ }_{u}, l_{u}$ and $j_{u}$. The variable leader $u$ indicates whether $u$ is still a leader contender. When the protocol has reached phase $_{u}=16$, it concludes by setting leaderDone ${ }_{u}$ to TRUE. To get an estimate of $\log n$, it uses the level $_{u}$ variable from the JUNTA process.

The protocol then runs in multiple phases. In even phases, all leader contender agents sample $\Theta(\log n)$ random bits. In odd phases, the maximum of the numbers represented by these random bits is sent to all agents via one-way epidemics. If a leader contender $u$ observes a larger value in the broadcast, it becomes a follower by setting leader ${ }_{u}$ to FALSE. The protocol is specified in Algorithm 8

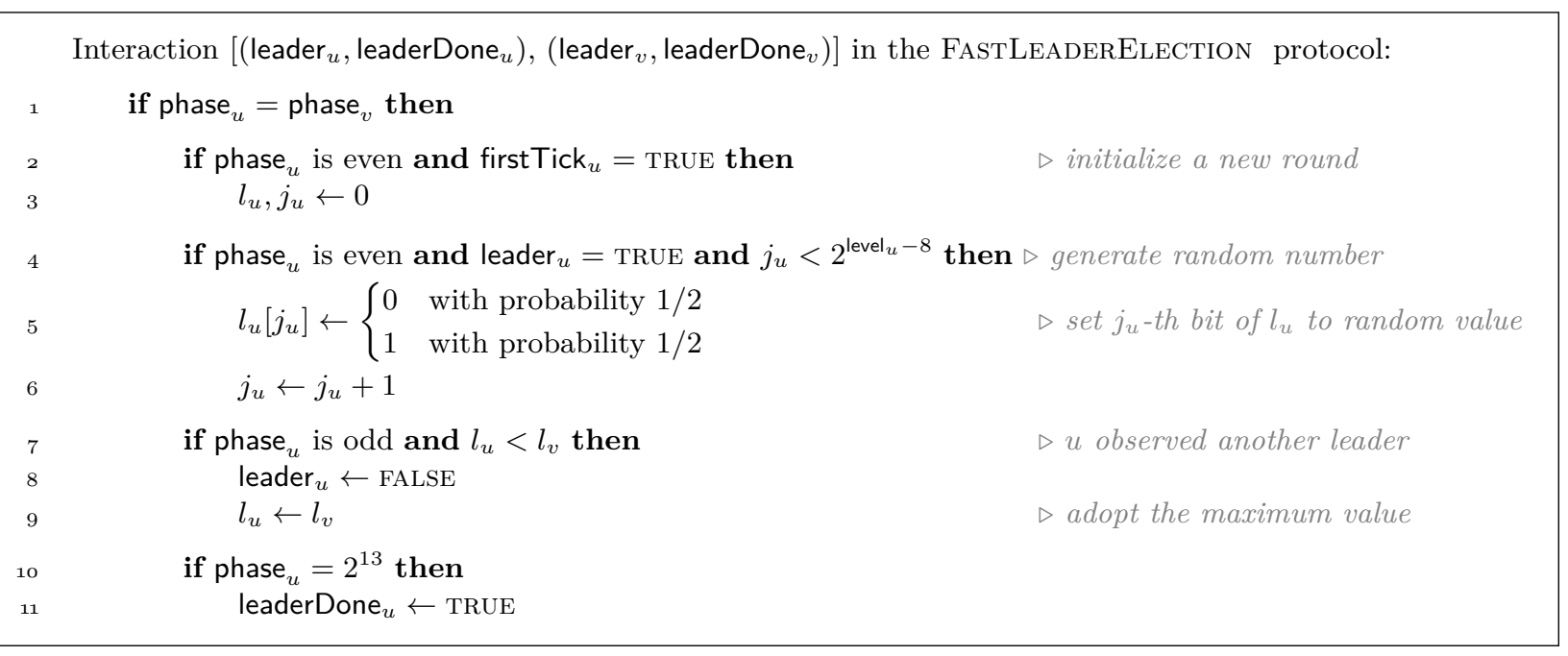

Algorithm 8: Protocol FastLeaderElection used in Algorithm 3

We now show Lemma 7 from Section 2.

Lemma 7 ([8]). The uniform protocol FASTLEADERELECTION elects a unique leader. It stabilizes in $\mathrm{O}(n \log n)$ interactions, using $\tilde{\mathrm{O}}(n)$ many states, w.h.p. Furthermore, after at most $\mathrm{O}\left(n \log ^{2} n\right)$ interactions all agents have leaderDone ${ }_{u}$ set to TRUE w.h.p.

Proof. First, observe that the protocol runs in constantly many phases. Therefore, every agent sets its flag leaderDone to TRUE after at most $\mathrm{O}(n \log n)$ interactions w.h.p.

Secondly, observe that there is always at least one leader contender. For every phase $t$, let $L(t)$ be the set of agents $u$ which have sampled the maximum value $l_{u}$. By definition, $L(t)$ is non-empty. Any contender can only become a follower in Line 8, and due to that rule it is impossible that any agent in the set $L(t)$ becomes a follower. Assume towards a contradiction that an agent $w$ in $L(t)$ becomes a follower. This can only occur if $l_{w}<l_{v}$ in some interaction with another agent $v$. However, this is a contradiction to $w \in L(t)$, the set of agents $u$ with largest value $l_{u}$.

Now consider an arbitrary but fixed pair of agents $(u, v)$. If $u$ and $v$ do not sample precisely the same bits in every even phase, at least one of them will become a follower in the next odd phase. The probability $p$ that both agents sample the same sequence of bits during the entire course of the protocol is

$$
p=1 / 2^{2^{13} \cdot 2^{\text {level }_{u}-8}} .
$$

For the maximal junta level level* in the modified junta process it holds that level* $\geq \log \log n-8$ w.h.p., see Lemma 4. We therefore get that

$$
p \leq 1 / 2^{2^{13} \cdot 2^{\log \log n-8}}=1 / n^{2} .
$$


We now use the above observation that there is always at least one leader contender. Let $u$ be a leader contender that concludes the FASTLEADERELECTION protocol by setting leaderDone ${ }_{u}$ to TRUE. We take the union bound over all other agents $v \neq u$ and obtain that with probably at most $1 / n$ there exists a second leader contender.

\section{E. Omitted Proofs from Section 4}

Proof of Lemma 11. At the end of Phase 0, w.h.p. all agents know $k_{u}$ from the leader according to one-way epidemics. At the end of Phase 1, w.h.p. $C \cdot 2^{k_{u}}$ tokens have been balanced such that $l_{v}=\Theta(1)$ for any agent $v$. At the end of Phase 2 , a total of at least $M \geq 4 \cdot n^{2}$ tokens have been balanced and every agent $v$ has load $l_{v}=\left[C \cdot 2^{2 k_{u}} / n\right\rceil \pm 1$ w.h.p.

We now need to show that every agent $v$ can output $n$ using its output function $\omega(v)=\left|C 2^{2 k_{v}} / l_{v}\right\rangle$, provided that $v$ knows the value $k_{v}=k_{u}=\log n \pm 3$ from the leader and the total load $M$ is at least $M=C \cdot 2^{2 k_{u}} \geq 4 n^{2}$ tokens. The proof is based on the proof of Lemma 3.8 from [15].

Observe that after the load balancing, w.h.p. $l_{v}=C \cdot 2^{2 k_{u}} / n+r$ where $r \in[-1.5,1.5]$ (the error term $r$ covers the remaining discrepancy plus 0.5 for the rounding error). Let $\hat{\omega}(v)$ be the output of agent $v$ before the rounding is applied. We derive, analogously to [15],

$$
\hat{\omega}(v)=\frac{M}{l_{v}}=\frac{C \cdot 2^{2 k_{u}}}{C \cdot 2^{2 k_{u}} / n+r}=\frac{C \cdot 2^{2 k_{u}} \cdot n}{C \cdot 2^{2 k_{u}}+r n}=\frac{C \cdot 2^{2 k_{u}} \cdot n}{C \cdot 2^{2 k_{u}}\left(1+\frac{r n}{C \cdot 2^{2 k_{u}}}\right)}=\frac{n}{1+\frac{r n}{C \cdot 2^{2 k_{u}}}}
$$

which gives us, since $r \in[-1.5,1.5]$

$$
\begin{aligned}
& \hat{\omega}(v) \geq \frac{n}{1+\frac{1.5 n}{C \cdot 2^{2 k u}}} \stackrel{\left(M \geq 4 n^{2}\right)}{\geq} \frac{n}{1+\frac{1.5 n}{4 n^{2}}}=\frac{n}{1+\frac{1}{3 n}}=n-\left(\frac{1}{3}-\frac{1}{3(3 n+1)}\right)>n-\frac{1}{2} \\
& \hat{\omega}(v) \leq \frac{n}{1-\frac{1.5 n}{C \cdot 2^{2 k} u}} \stackrel{\left(M \geq 4 n^{2}\right)}{\leq} \frac{n}{1-\frac{1.5 n}{4 n^{2}}}=\frac{n}{1-\frac{1}{3 n}}=n+\frac{1}{3}+\frac{1}{3(3 n-1)}<n+\frac{1}{2} .
\end{aligned}
$$

Therefore, $\omega(v)=\lfloor\hat{\omega}(v)\rceil=n$.

\section{F. Stable Exact Counting}

In this appendix we describe how to build a stable protocol to count the exact population size. We use a similar approach as in Section 3. We check for errors during the course of the protocol, and if we detect an error, the interacting agents raise an error flag. This error flag is sent to all other agents via one-way epidemics. Every agent which has set the error flag performs a simple backup protocol described in Appendix $\mathrm{C}$ that outputs the correct result with probability 1 after $\mathrm{O}\left(n^{2} \log n\right)$ interactions w.h.p.

We first describe which error detection mechanisms are additionally required for Algorithm 3 and then we show that the combined protocol stabilizes.

For the FAstLeaderElection protocol (see Appendix D) we know that there is always at least one leader. If, however, more than two leaders conclude the leader election protocol, this error can be detected whenever they directly interact with each other. In this case they both raise the error flag.

The second type of error we detect during the course of the protocol are synchronization problems with the phase clock. We assume that all agents count the exact number of phases (until the last 
phase of the REFinementStage protocol). When now two agents interact that have a different phase counter, both agents raise the error flag.

Finally, in the ReFinementStage protocol all agents $v$ verify in Line 6 that they have a load of at least $l_{v} \geq 2^{5}$ before multiplying their load with $2^{k_{v}}$. At the same time, all agents start to compare their $k_{v}$ values. If any agent does not have sufficiently many tokens, or if two agents interact with different $k_{v}$ values, the agents raise the error flag.

We now give the full proof for Theorem 2, which is re-stated for completeness as follows.

Theorem 2. The protocol COUnTEXACT is uniform and outputs the exact population size $n$. It stabilizes in $\mathrm{O}(n \log n)$ interactions and uses $\tilde{\mathrm{O}}(n)$ states, w.h.p.

Proof. There is always at least one leader. If, however, multiple leaders conclude the FASTLEADEREleCtion protocol, this error is detected and the protocol stabilizes due to the backup protocol. We therefore consider in the following only the case when precisely one leader concludes the FASTLEADERELECTION protocol.

Note that In the way we elect the junta there is always at least one junta agent. Therefore the phase clocks perform an infinite sequence of ticks with probability 1 . However, we cannot guarantee that all agents are always in exactly the same phase w.r.t. their ticks, and the length of the phases might diverge drastically from the expectation (albeit only with low probability). Now since all agents count the exact number of phases, whenever two agents interact that have a different phase counter, both agents raise the error flag and the protocol stabilizes due to the backup protocol.

The presence of a unique leader guarantees that in the ApproximationStAGE protocol the total load is initially at least 1 . Recall that the phase clock ticks with probability 1 , even if the agents are not properly synchronized. Therefore, the load grows in the ApproximationStage protocol over

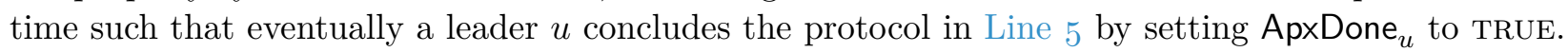
Therefore, with probability 1 the leader starts the REFinementStaGe protocol by setting its load $l_{u}$ to $C \cdot 2^{k_{u}}$. In the REFinementSTAGE protocol all agents $v$ verify that they have a load of at least $l_{v} \geq 2^{5}-1.5$ before multiplying their load with $2^{k_{v}}$. If this is the case for every agent, the value $k_{u}$ from the leader $u$ has been at least $\log n-3$, and the total load is at least $4 n^{2}$ as required in the proof of Lemma 11. Otherwise, the under-loaded agents raise the error flag since the total load is insufficient to exactly compute $n$. Again, the protocol stabilizes due to the backup protocol. If all agents have passed this last error check in Phase 2 of the REFinementStage protocol (and all agents $v$ hold the same value $k_{v}$ ), then all agents will eventually output $n$ with probability 1 . Otherwise, at least one agent $v$ is in the error state (or will enter the error state when comparing its $k_{v}$ value) and thus with probability 1 all agents will eventually output $n$ via the backup protocol.

It remains to argue the required number of states and the time until the protocol stabilizes.

For the required number of states, observe that for each agent $u$ w.h.p. level ${ }_{u}=\mathrm{O}(\log \log n)$ (see Lemma 4), $i_{u}=\mathrm{O}(1)$ and $k_{u} \leq \log n+3$ (see Lemma 10), and thus $l_{u}$ holds at most $\mathrm{O}(n)$ tokens in the RefinementStage protocol. The total number of states is therefore w.h.p. at most $\mathrm{O}(\log \log n \cdot \log n \cdot n)$.

The protocol stabilizes once all agents have concluded the second phase of the REFInEMENTSTAGE protocol. The FAStLEAderElection protocol requires w.h.p. at most $\mathrm{O}(n \log n)$ interactions (see Lemma 7), the ApproximationStage protocol also requires w.h.p. at most $\mathrm{O}(n \log n)$ interactions (see Lemma 10), and the RefinementStage protocol requires 3 phases, i.e., $\mathrm{O}(n \log n)$ interactions, as well. Therefore the protocol CountExaCt stabilizes within $\mathrm{O}(n \log n)$ interactions w.h.p. 